\title{
A Semismooth Newton Method for Nonlinear Parameter Identification Problems with Impulsive Noise*
}

\author{
Christian Clason ${ }^{\dagger}$ and Bangti Jin
}

\begin{abstract}
This work is concerned with nonlinear parameter identification in partial differential equations subject to impulsive noise. To cope with the non-Gaussian nature of the noise, we consider a model with $\mathrm{L}^{1}$ fitting. However, the nonsmoothness of the problem makes its efficient numerical solution challenging. By approximating this problem using a family of smoothed functionals, a semismooth Newton method becomes applicable. In particular, its superlinear convergence is proved under a second-order condition. The convergence of the solution to the approximating problem as the smoothing parameter goes to zero is shown. A strategy for adaptively selecting the regularization parameter based on a balancing principle is suggested. The efficiency of the method is illustrated on several benchmark inverse problems of recovering coefficients in elliptic differential equations, for which one- and two-dimensional numerical examples are presented.
\end{abstract}

Key words. semismooth Newton method, parameter identification, impulsive noise, $\mathrm{L}^{1}$ data fitting, regularization parameter, noise model

AMS subject classifications. 65M32, 65J20, 49M15, 49M29

DOI. $10.1137 / 110826187$

1. Introduction. We are interested in the nonlinear inverse problem

$$
S(u)=y^{\delta},
$$

where $S: \mathcal{X} \rightarrow \mathcal{Y}$ is the parameter-to-observation mapping and $y^{\delta}$ represents experimental measurements corrupted by impulsive noise. Throughout we assume that the space $\mathcal{Y}$ compactly embeds into $\mathrm{L}^{q}$ for some $q>2, y^{\delta}$ is bounded almost everywhere, and $\mathcal{X}$ is a Hilbert space. The spaces $\mathcal{X}$ and $\mathcal{Y}$ are defined on the bounded domains $\omega \subset \mathbb{R}^{n}$ and $D \subset \mathbb{R}^{m}$, respectively. Such models arise naturally in distributed parameter identification for differential equations, where typically $\mathcal{Y}$ is $\mathrm{H}^{1}(D)$ or $\mathrm{H}^{\frac{1}{2}}(D)$ and $\mathcal{X}$ is $\mathrm{L}^{2}(\omega)$ or $\mathrm{H}^{1}(\omega)[3]$.

The noise model for the measured data $y^{\delta}$ plays a critical role in formulating and solving the problem. In practice, an additive Gaussian noise model is customarily adopted, which leads to the standard $\mathrm{L}^{2}$ fitting. However, non-Gaussian (e.g., Laplace or Cauchy) noise - which admits the presence of significant outliers - may also occur. An extreme case is impulsive noise such as

\footnotetext{
${ }^{*}$ Received by the editors February 28, 2011; accepted for publication (in revised form) December 12, 2011; published electronically April 12, 2012.

http://www.siam.org/journals/siims/5-2/82618.html

${ }^{\dagger}$ Institute for Mathematics and Scientific Computing, University of Graz, Heinrichstrasse 36, A-8010 Graz, Austria (christian.clason@uni-graz.at). This author's work was supported by the Austrian Science Fund (FWF) under grant SFB F32 (SFB "Mathematical Optimization and Applications in Biomedical Sciences").

${ }^{\ddagger}$ Department of Mathematics and Institute for Applied Mathematics and Computational Science, Texas A\&M University, College Station, TX 77843-3368 (btjin@math.tamu.edu). This author's work was supported by Award KUS-C1-016-04, made by King Abdullah University of Science and Technology (KAUST).
} 
salt-and-pepper or random-valued noise, which frequently occurs in digital image acquisition and processing due to, e.g., malfunctioning pixels in camera sensors, faulty memory locations in hardware, or transmission in noisy channels [6]. Before giving a formal definition below (section 1.2), let us briefly describe its salient feature and motivate the use of $\mathrm{L}^{1}$ fitting. The impulsive noise models considered here are characterized by the fact that only a (possibly large) number of points are subject to large errors, while the remaining data points stay intact. (In effect, such noise is "outliers only.") Such noise thus has a sparsity property. Since it is well known that $\mathrm{L}^{1}$ norms as penalties promote sparse solutions [10,44], the expectation of a sparse residual quite naturally leads to $\mathrm{L}^{1}$ fitting. In contrast, $\mathrm{L}^{2}$ fitting assumes that all points are corrupted by independent and identically distributed Gaussian noise, and one single outlier can exert substantial influence on the reconstruction [22].

These considerations motivate adopting the model

$$
\min _{u \in U}\left\|S(u)-y^{\delta}\right\|_{\mathrm{L}^{1}}+\frac{\alpha}{2}\|u\|_{\mathcal{X}}^{2},
$$

where the set $U \subset \mathcal{X}$ is convex and closed, representing physical constraints on the unknown $u$. We are mainly interested in various structural properties of the $\mathrm{L}^{1}$-norm fitting compared with the more conventional $\mathrm{L}^{2}$-norm counterpart. Our main goal in this work is to resolve the computational obstacles posed by the nondifferentiability of the $\mathrm{L}^{1}$-norm and nonlinearity of the operator $S$, such that Newton-type methods are applicable when the operator $S$ has the necessary differentiability properties.

Due to the practical significance of $\mathrm{L}^{1}$ models, there has been a growing interest in analyzing their properties and in developing efficient minimization algorithms, e.g., in imaging $[15,34]$ as well as parameter identification [8]. A number of recent works have addressed the analytical properties of models with $\mathrm{L}^{1}$ fitting, explaining their superior performance over the standard model for certain types of noise and elaborating the geometrical structure of the minimizers in the context of image denoising $[2,9,17,50]$, i.e., when $S$ is the identity operator. In addition, several efficient algorithms $[14,15,16,48]$ have been developed for such problems.

However, all these works are concerned only with linear inverse problems, and their analysis and algorithms are not directly applicable to the nonlinear case of our interest. The optimality system is not differentiable in a generalized sense and thus cannot be solved directly with a (semismooth) Newton method. We consider a smoothed variant and prove the convergence as the smoothing parameter tends to zero. The smoothed optimality system is solved by a semismooth Newton (SSN) method, and its superlinear local convergence is established under a second-order condition. To the best of our knowledge, this work represents a first investigation on $\mathrm{L}^{1}$ fitting with general nonlinear inverse problems. The applicability of the proposed approach and its numerical performance are illustrated with several benchmark problems for distributed parameter identification for elliptic partial differential equations.

The rest of this work is organized as follows. In the remainder of this section, we introduce a selection of model problems for which our approach is applicable (section 1.1) and state a precise definition of the considered noise models (section 1.2). In section 2, we discuss wellposedness and regularization properties for nonlinear $\mathrm{L}^{1}$ fitting (section 2.1) and derive the optimality system (section 2.2). The approximating problem, its convergence as the smoothing parameter tends to zero, and its numerical solution using an SSN method are studied in sec- 
tion 3. We also discuss the important issue of choosing suitable regularization and smoothing parameters. Finally, in section 4, we present numerical results for our model problems.

1.1. Model problems. In this part, we describe three nonlinear model problems - an inverse potential problem, an inverse Robin coefficient problem, and an inverse diffusion coefficient problem - for which our SSN method is applicable.

Inverse potential problem. A first nonlinear model problem consists in recovering the potential term in an elliptic equation. Let $\Omega \subset \mathbb{R}^{d}$ be an open bounded domain with a Lipschitz boundary $\Gamma$. We consider the equation

$$
\left\{\begin{aligned}
-\Delta y+u y & =f & & \text { in } \Omega, \\
\frac{\partial y}{\partial n} & =0 & & \text { on } \Gamma .
\end{aligned}\right.
$$

The inverse problem is to recover the potential $u$ defined on $\omega=\Omega$ from noisy observational data $y^{\delta}$ in the domain $D=\Omega$, i.e., $S$ maps $u \in \mathcal{X}=\mathrm{L}^{2}(\Omega)$ to the solution $y \in \mathcal{Y}=\mathrm{H}^{1}(\Omega)$ of (1.1). Such problems arise in heat transfer, e.g., damping design [43] and identifying heat radiative coefficient [47]. We shall seek $u$ in the admissible set $U=\left\{u \in \mathrm{L}^{\infty}(\Omega): u \geq c\right\} \subset \mathcal{X}$ for some fixed $c>0$.

Inverse Robin coefficient problem. Our second example considers the recovery of a Robin boundary condition from boundary observation. Let $\Omega \subset \mathbb{R}^{2}$ be an open bounded domain with a Lipschitz boundary $\Gamma$ consisting of two disjoint parts $\Gamma_{i}$ and $\Gamma_{c}$. We consider the equation

$$
\left\{\begin{aligned}
-\Delta y & =0 & & \text { in } \Omega \\
\frac{\partial y}{\partial n} & =f & & \text { on } \Gamma_{c} \\
\frac{\partial y}{\partial n}+u y & =0 & & \text { on } \Gamma_{i}
\end{aligned}\right.
$$

The inverse problem consists in recovering the Robin coefficient $u$ defined on $\omega=\Gamma_{i}$ from noisy observational data $y^{\delta}$ on the boundary $D=\Gamma_{c}$, i.e., $S$ maps $u \in \mathcal{X}=\mathrm{L}^{2}\left(\Gamma_{i}\right)$ to $\left.y\right|_{\Gamma_{c}} \in \mathcal{Y}=\mathrm{H}^{\frac{1}{2}}\left(\Gamma_{c}\right)$, where $\left.v \mapsto v\right|_{\Gamma_{c}}$ denotes the Dirichlet trace operator and $y$ is the solution to (1.2). This class of problems arises in corrosion detection and thermal analysis of quenching processes $[8,33]$. We shall seek $u$ in the admissible set $U=\left\{u \in \mathrm{L}^{\infty}\left(\Gamma_{i}\right): u \geq c\right\} \subset \mathcal{X}$ for some fixed $c>0$.

Inverse diffusion coefficient problem. Our last example, identification of a diffusion coefficient, addresses stronger regularization for the parameter. Let $\Omega \subset \mathbb{R}^{2}$ be an open bounded domain with a smooth boundary $\Gamma$. We consider the equation

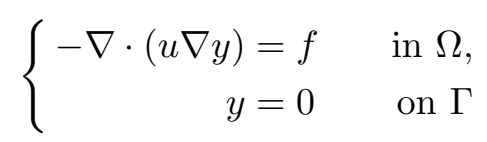

with $f \in \mathrm{L}^{q}(\Omega)$ for some $q>2$. The inverse problem consists in recovering the diffusion coefficient $u$ within $\omega=\Omega$ from the noisy observational data $y^{\delta}$ in the domain $D=\Omega$, i.e., $S$ maps $u \in \mathcal{X}=\mathrm{H}^{1}(\Omega)$ to the solution $y \in \mathcal{Y}=\mathrm{W}_{0}^{1, q}(\Omega), q>2$, of (1.3). Such problems 
arise in estimating the permeability of underground flow and the conductivity of heat transfer $[3,12,49]$. We shall seek $u$ in the admissible set $U=\left\{u \in \mathrm{L}^{\infty}(\Omega): \lambda \leq u \leq \lambda^{-1}\right\} \cap \mathcal{X}$ for some fixed $\lambda \in(0,1)$.

These model problems share the following properties, which are verified in Appendix A and are sufficient to guarantee the applicability of our approach:

(A1) The operator $S$ is uniformly bounded in $U \subset \mathcal{X}$ and completely continuous: If for $u \in U$, the sequence $\left\{u_{n}\right\} \subset U$ satisfies $u_{n} \rightarrow u$ in $\mathcal{X}$, then

$$
S\left(u_{n}\right) \rightarrow S(u) \quad \text { in } \mathrm{L}^{2}(D) .
$$

(A2) $S$ is twice Fréchet differentiable.

(A3) There exists a constant $C>0$ such that for all $u \in U$ and $h \in \mathcal{X}$ there holds

$$
\left\|S^{\prime}(u) h\right\|_{\mathrm{L}^{2}} \leq C\|h\|_{\mathcal{X}}
$$

(A4) there holds

$$
\left\|S^{\prime \prime}(u)(h, h)\right\|_{\mathrm{L}^{2}} \leq C\|h\|_{\mathcal{X}}^{2} .
$$

The twice differentiability of $S$ in (A2) is required for a Newton method (see section 3.2) and ensures strict differentiability required for the chain rule; see the proof of Theorem 2.7. The a priori estimate in (A3) is employed in analyzing the convergence of the approximate solutions, while (A4) will be used to show local superlinear convergence of the SSN method.

1.2. Noise model. We now motivate the use of $\mathrm{L}^{1}$ fitting for impulsive noise from a statistical viewpoint (cf. $[22,24,28])$. The exact data $y^{\dagger}=S\left(u^{\dagger}\right)$, where $u^{\dagger}$ is the true solution, defined over a domain $D$, is corrupted by noise. The contaminated observation $y^{\delta}$ is formed pointwise by

$$
y^{\delta}(x)=f\left(y^{\dagger}, \xi_{r}\right)(x) \quad x \in D
$$

where $\xi_{r}(x)$ is a real-valued random variable, $r \in[0,1]$ is a noise parameter, and the function $f$ represents the noise formation mechanism. We assume that for any two distinct points $x_{1}, x_{2} \in$ $D, \xi_{r}\left(x_{1}\right)$ and $\xi_{r}\left(x_{2}\right)$ are independent. In practice, the Gaussian noise model (and hence $\mathrm{L}^{2}$ fitting) stands out predominantly. This is often justified by appealing to the celebrated central limit theorem: a Gaussian distribution is suitable for data that are formed as the sum of a large number of independent components [22]. Even in the absence of such justifications, this model is still often preferred due to its computational and analytical conveniences. However, it is also clear that not all real-world data can be adequately described by the Gaussian model. Here, we consider impulsive noise models: There exist (many) points $x \in D$ with $f\left(y, \xi_{r}\right)(x)=y(x)$. The two most common types of impulsive noises, e.g., arising in digital image processing [6], are salt-and-pepper noise and random-valued impulse noise (RVIN).

The salt-and-pepper noise model is especially common in image processing, and it reflects a wide variety of processes that result in the same image degradation: the corrupted data points (where $\xi_{r} \neq 0$ ) only take a fixed maximum ("salt") or minimum ("pepper") value. A 
simple model is as follows:

$$
y^{\delta}(x)= \begin{cases}y^{\dagger}(x), & \text { with probability } 1-r, \\ y_{\max }, & \text { with probability } \frac{r}{2}, \\ y_{\min }, & \text { with probability } \frac{r}{2},\end{cases}
$$

where $y_{\max }$ and $y_{\min }$ are the maximum and minimum of the signal, respectively, and the parameter $r \in(0,1)$ represents the percentage of corrupted data points.

In the context of parameter identification problems, it is more reasonable to allow arbitrary random values at the corrupted data points, which gives rise to the RVIN model

$$
y^{\delta}(x)= \begin{cases}y^{\dagger}(x) & \text { with probability } 1-r, \\ y^{\dagger}(x)+\xi(x) & \text { with probability } r\end{cases}
$$

where $\xi(x)$ is a random variable, e.g., normally distributed with mean zero and typically large variance. Clearly, RVIN is generated by the random variable $\xi(x)$ and reproduces the latter if $r=1$. However, its characteristic is fundamentally different from that of $\xi(x)$ for $r<1$ : there exist data points which are not corrupted by noise which carry a significant amount of information in the data.

Like many non-Gaussian noise models such as Laplace and Cauchy noise, impulsive noise features significant outliers, i.e., data points that lie far away from the bulk of the data. Statistically, this calls for robust methods (robust estimation in statistics [28]). One classical approach is to first identify the outliers with noise detectors, e.g., adaptive median filter, and then perform inversion/reconstruction on the data with outliers excluded. Its success relies crucially on accurate identification of all outliers, which remains very challenging in case of multiple outliers [42], and misidentification can significantly compromise the reconstruction. The $\mathrm{L}^{1}$ approach provides a more systematic strategy for handling outliers due to its ability to implicitly and accurately detect outliers and to automatically prune them from the inversion procedure. The use of $\mathrm{L}^{1}$ fitting has shown very promising results in a number of practical applications $[4,14,16]$. There have been some theoretical justifications of these empirical observations [27]. They are also reflected in the optimality system, where the dual variable acts as a noise detector (cf. Corollary 2.8). In contrast, $\mathrm{L}^{2}$ fitting tends to place equal weight on all data points and thus suffers from a lack of robustness: One single outlier can exert significant influences globally and may spoil the reconstruction completely [22, p. 443].

We observe that these statistical considerations are finite-dimensional in nature. Nonetheless, they directly motivate the use of the continuous analogue, the $\mathrm{L}^{1}$ model, for parameter identification problems. We would like to note that the model considered here remains deterministic, despite the preceding statistical motivations. In particular, we do not regard the observational data $y^{\delta}$ as an "impulsive" type of stochastic process in function spaces, instead seeing it only as a realization of such stochastic process, as is usually the case for deterministic inverse problems [19]. However, a stochastic analogue of the $\mathrm{L}^{1}$ model in function spaces is also of great interest. We recall that the more conventional Gaussian model in function spaces can be modeled as a Hilbert space-valued random variable - and more generally a Hilbert space process - whose properties are characterized by its covariance structure. (See the nice 
summary in [5, section 2.5].) It would be desirable to have analogous characterizations for the $\mathrm{L}^{1}$ model. Some results in this direction can be found in [37], where Besov priors were (formally) studied that might also be applied to impulsive noises.

2. $\mathrm{L}^{1}$ fitting for nonlinear inverse problems. The above considerations motivate considering the problem

$$
\min _{u \in U}\left\{\mathcal{J}_{\alpha}(u) \equiv\left\|S(u)-y^{\delta}\right\|_{\mathrm{L}^{1}}+\frac{\alpha}{2}\left\|u-u_{0}\right\|_{\mathcal{X}}^{2}\right\}
$$

for the nonlinear operator $S: U \subset \mathcal{X} \rightarrow \mathcal{Y}$ satisfying assumptions (A1)-(A4) (although the results of this and the next section require only (A1) and (A2)) and given $y^{\delta} \in \mathrm{L}^{\infty}(D)$. Here, $u_{0}$ is an initial guess which also plays the role of a selection criterion.

2.1. Existence and regularization properties. We first address the well-posedness of the problem $(\mathcal{P})$. In this section, we shall denote a minimizer of the functional $\mathcal{J}_{\alpha}$ by $u_{\alpha}^{\delta}$, while $u_{\alpha}$ will be a minimizer with $y^{\delta}$ replaced by the exact data $y^{\dagger}$. We assume that $y^{\dagger}$ is attainable, i.e., that there exists an element $u^{\dagger} \in U$ such that $y^{\dagger}=S\left(u^{\dagger}\right)$. If $u^{\dagger}$ is not unique, it always refers to a $u_{0}$-minimum-norm solution, i.e., an element minimizing $\left\|u-u_{0}\right\|_{\mathcal{X}}$ over the set of solutions to $S(u)=y^{\dagger}$. Throughout, $C$ denotes a generic constant, whose value may differ at different occurrences.

The proof of the next result is standard (see, e.g., [20], [19, Chap. 10]) and is thus omitted.

Theorem 2.1. Under Assumption (A1), problem $(\mathcal{P})$ is well-posed and consistent, i.e.,

(i) there exists at least one minimizer $u_{\alpha}^{\delta} \in U$ to problem $(\mathcal{P})$;

(ii) for a sequence of data $\left\{y_{n}\right\}$ such that $y_{n} \rightarrow y^{\delta}$ in $\mathrm{L}^{1}(D)$, the sequence $\left\{u_{\alpha}^{n}\right\}$ of minimizers contains a subsequence converging to $u_{\alpha}^{\delta}$;

(iii) if the regularization parameter $\alpha=\alpha(\delta)$ satisfies

$$
\lim _{\delta \rightarrow 0} \alpha(\delta)=\lim _{\delta \rightarrow 0} \frac{\delta}{\alpha(\delta)}=0
$$

then the sequence $\left\{u_{\alpha(\delta)}^{\delta}\right\}$ has a subsequence converging to $u^{\dagger}$ as $\delta \rightarrow 0$.

If we assume Lipschitz continuity of the derivative $S^{\prime}$ and a (standard) source condition, we have the following result on the convergence rate for the a priori parameter choice rule $\alpha=\alpha(\delta) \sim \delta^{\varepsilon}$ for any $\varepsilon \in(0,1)$ (cf. [26, 40]).

Theorem 2.2. Let $y^{\delta} \in \mathcal{Y}$ satisfy $\left\|y^{\delta}-y^{\dagger}\right\|_{\mathrm{L}^{1}} \leq \delta$ and let $u^{\dagger} \in U$ be a $u_{0}$-minimum norm solution of $S(u)=y^{\dagger}$. Moreover, let the following conditions be fulfilled:

(i) There exists an $L>0$ such that $\left\|S^{\prime}\left(u^{\dagger}\right)-S^{\prime}(z)\right\|_{\mathrm{L}^{2}} \leq L\left\|u^{\dagger}-z\right\|_{\mathcal{X}}$ for all $z \in U \subset \mathcal{X}$.

(ii) There exists a $w \in \mathrm{L}^{\infty}(D) \cap \mathrm{L}^{2}(D)$ with $L\|w\|_{\mathrm{L}^{2}}<1$ satisfying $u^{\dagger}-u_{0}=S^{\prime}\left(u^{\dagger}\right)^{*} w$.

Then for any fixed $\varepsilon \in(0,1)$, the choice $\alpha \sim \delta^{\varepsilon}$ and $\delta$ sufficiently small, we have the estimate

$$
\left\|u_{\alpha}^{\delta}-u^{\dagger}\right\|_{\mathcal{X}} \leq C \delta^{\frac{1-\varepsilon}{2}} .
$$

Proof. By the minimizing property of $u_{\alpha}^{\delta}$ and $\left\|y^{\delta}-y^{\dagger}\right\|_{\mathrm{L}^{1}} \leq \delta$, we obtain

$$
\left\|S\left(u_{\alpha}^{\delta}\right)-y^{\delta}\right\|_{L^{1}}+\frac{\alpha}{2}\left\|u_{\alpha}^{\delta}-u_{0}\right\|_{\mathcal{X}}^{2} \leq \delta+\frac{\alpha}{2}\left\|u^{\dagger}-u_{0}\right\|_{\mathcal{X}}^{2}
$$


and hence

$$
\left\|S\left(u_{\alpha}^{\delta}\right)-y^{\delta}\right\|_{L^{1}}+\frac{\alpha}{2}\left\|u_{\alpha}^{\delta}-u^{\dagger}\right\|_{\mathcal{X}}^{2} \leq \delta+\alpha\left\langle u^{\dagger}-u_{0}, u^{\dagger}-u_{\alpha}^{\delta}\right\rangle_{\mathcal{X}}
$$

Now by the source condition (ii), we obtain

$$
\left\|S\left(u_{\alpha}^{\delta}\right)-y^{\delta}\right\|_{L^{1}}+\frac{\alpha}{2}\left\|u_{\alpha}^{\delta}-u^{\dagger}\right\|_{\mathcal{X}}^{2} \leq \delta+\alpha\left\langle w, S^{\prime}\left(u^{\dagger}\right)\left(u^{\dagger}-u_{\alpha}^{\delta}\right)\right\rangle_{\mathrm{L}^{2}} .
$$

The Fréchet differentiability of $S$ and condition (i) imply

$$
S\left(u_{\alpha}^{\delta}\right)=S\left(u^{\dagger}\right)+S^{\prime}\left(u^{\dagger}\right)\left(u_{\alpha}^{\delta}-u^{\dagger}\right)+r\left(u_{\alpha}^{\delta}, u^{\dagger}\right)
$$

with $\left\|r\left(u_{\alpha}^{\delta}, u^{\dagger}\right)\right\|_{L^{2}} \leq \frac{L}{2}\left\|u_{\alpha}^{\delta}-u^{\dagger}\right\|_{\mathcal{X}}^{2}$. Combining these estimates leads to

$$
\begin{aligned}
\left\|S\left(u_{\alpha}^{\delta}\right)-y^{\delta}\right\|_{\mathrm{L}^{1}}+\frac{\alpha}{2}\left\|u_{\alpha}^{\delta}-u^{\dagger}\right\|_{\mathcal{X}}^{2} \leq & \delta+\alpha\left\langle w,\left(y^{\dagger}-y^{\delta}\right)+\left(y^{\delta}-S\left(u_{\alpha}^{\delta}\right)\right)+r\left(u_{\alpha}^{\delta}, u^{\dagger}\right)\right\rangle_{\mathrm{L}^{2}} \\
\leq & \delta+\alpha\|w\|_{\mathrm{L}^{\infty}} \delta+\alpha\|w\|_{\mathrm{L}^{\infty}}\left\|S\left(u_{\alpha}^{\delta}\right)-y^{\delta}\right\|_{\mathrm{L}^{1}} \\
& +\frac{\alpha}{2} L\|w\|_{\mathrm{L}^{2}}\left\|u_{\alpha}^{\delta}-u^{\dagger}\right\|_{\mathcal{X}}^{2}
\end{aligned}
$$

and hence

$$
\left(1-\alpha\|w\|_{\mathrm{L}^{\infty}}\right)\left\|S\left(u_{\alpha}^{\delta}\right)-y^{\delta}\right\|_{\mathrm{L}^{1}}+\frac{\alpha}{2}\left(1-L\|w\|_{\mathrm{L}^{2}}\right)\left\|u_{\alpha}^{\delta}-u^{\dagger}\right\|_{\mathcal{X}}^{2} \leq \delta+\alpha\|w\|_{\mathrm{L}^{\infty}} \delta .
$$

Now the desired result follows from the condition $L\|w\|_{\mathrm{L}^{2}}<1$ and the choice of $\alpha$ such that $\alpha\|w\|_{L^{\infty}}<1$ for $\delta$ sufficiently small.

Remark 2.3. An inspection of the proof shows that a rate of order $\mathcal{O}\left(\delta^{\frac{1}{2}}\right)$ can be achieved for a choice rule $\alpha(\delta)$ for which the limit $\alpha^{*}=\lim _{\delta \rightarrow 0} \alpha(\delta)$ satisfies $\alpha^{*}<1 /\|w\|_{L^{\infty}}$ and $\alpha^{*}>0$. We point out that the source condition $u^{\dagger}-u_{0}=S^{\prime}\left(u^{\dagger}\right)^{*} w$ might be further relaxed by utilizing the structure of the adjoint operator; see [29] for relevant discussions in the context of parameter identification.

The a priori choice gives only an order of magnitude for $\alpha$ and is thus practically inconvenient to use. In contrast, the discrepancy principle [32, 39] enables constructing a concrete scheme for determining the regularization parameter $\alpha$. Specifically, one chooses $\alpha=\alpha(\delta)$ such that

$$
\left\|S\left(u_{\alpha}^{\delta}\right)-y^{\delta}\right\|_{\mathrm{L}^{1}}=c \delta,
$$

where $c \geq 1$ is a constant. Numerically, it can be realized efficiently by either a two-parameter algorithm based on model functions or the secant method [32], but it requires knowledge of the noise level $\delta$.

The next result shows a $\mathcal{O}\left(\delta^{\frac{1}{2}}\right)$ convergence rate. Its proof is almost identical to that of Theorem 2.2 (cf. [32]) and hence is omitted.

Theorem 2.4 (discrepancy principle). Let conditions (i)-(ii) in Theorem 2.2 be fulfilled. Then for the choice $\alpha$ determined by (2.1), there holds

$$
\left\|u_{\alpha}^{\delta}-u^{\dagger}\right\|_{\mathcal{X}} \leq C \delta^{\frac{1}{2}}
$$


The next result shows an interesting property of $\mathrm{L}^{1}$ fitting (and in general, of one-homogeneous discrepancy functionals; cf. [7]) in the case of exact data: the regularized solution $u_{\alpha}$ coincides with the exact solution $u^{\dagger}$ if the regularization parameter $\alpha$ is sufficiently small. This is in sharp contrast to quadratic $\mathrm{L}^{2}$ fitting, where the Tikhonov minimizer is different from the true solution for every $\alpha>0$.

Theorem 2.5 (exact recovery). Let conditions (i) and (ii) in Theorem 2.2 be fulfilled. Then, $u_{\alpha}=u^{\dagger}$ holds for $\alpha>0$ sufficiently small.

Proof. We only sketch the proof. By the minimizing properties of $u_{\alpha}$ and the source condition, we arrive at

$$
\left\|S\left(u_{\alpha}\right)-y^{\dagger}\right\|_{\mathrm{L}^{1}}+\frac{\alpha}{2}\left\|u_{\alpha}-u^{\dagger}\right\|_{\mathcal{X}}^{2} \leq-\alpha\left\langle w, S^{\prime}\left(u^{\dagger}\right)\left(u_{\alpha}-u^{\dagger}\right)\right\rangle_{\mathrm{L}^{2}} .
$$

As before, we obtain by the Fréchet differentiability of $S$ that

$$
\begin{aligned}
\left\|S\left(u_{\alpha}\right)-y^{\dagger}\right\|_{\mathrm{L}^{1}}+\frac{\alpha}{2}\left\|u_{\alpha}-u^{\dagger}\right\|_{\mathcal{X}}^{2} & \leq \alpha\left\langle w,\left(y^{\dagger}-S\left(u_{\alpha}\right)\right)+r\left(u_{\alpha}, u^{\dagger}\right)\right\rangle_{\mathrm{L}^{2}} \\
& \leq \alpha\|w\|_{\mathrm{L}^{\infty}}\left\|S\left(u_{\alpha}\right)-y^{\dagger}\right\|_{\mathrm{L}^{1}}+\frac{\alpha}{2} L\|w\|_{\mathrm{L}^{2}}\left\|u_{\alpha}-u^{\dagger}\right\|_{\mathcal{X}}^{2} .
\end{aligned}
$$

Hence, for $\alpha \leq 1 /\|w\|_{L^{\infty}}$, we have $\left\|u_{\alpha}-u^{\dagger}\right\|_{\mathcal{X}}=0$, i.e., $u_{\alpha}=u^{\dagger}$.

2.2. Optimality system. We next derive the necessary first-order optimality conditions for $u_{\alpha}:=u_{\alpha}^{\delta}$ (slightly abusing the notation).

Remark 2.6. In this work, we assume that the true solution $u^{\dagger}$ of the inverse problem (and a minimizer $u_{\alpha}$ of $(\mathcal{P})$ ) lies in the interior $U_{\text {int }}$ of $U$ and we do not explicitly enforce the constraint $u \in U$, in order to focus the presentation on the treatment of the nonsmoothness inherent in the $\mathrm{L}^{1}$-fitting problem. There is no fundamental difficulty in including this constraint in the optimization, however, in which case the first equality in the optimality conditions (OS) should be replaced by a variational inequality. When the domain of definition is given by box constraints (as in the model problems), the modified optimality system can still be solved using an SSN method after applying a Moreau-Yosida regularization; cf. [23].

Theorem 2.7. For any local minimizer $u_{\alpha} \in U_{\text {int }} \subset \mathcal{X}$ of problem $(\mathcal{P})$ there exists a $p_{\alpha} \in$ $\mathrm{L}^{\infty}(D)$ with $\left\|p_{\alpha}\right\|_{\mathrm{L}^{\infty}} \leq 1$ such that the following relations hold:

$$
\left\{\begin{aligned}
S^{\prime}\left(u_{\alpha}\right)^{*} p_{\alpha}+\alpha j\left(u_{\alpha}-u_{0}\right) & =0, \\
\left\langle S\left(u_{\alpha}\right)-y^{\delta}, p-p_{\alpha}\right\rangle_{\mathrm{L}^{2}} & \leq 0 \quad \text { for all }\|p\|_{\mathrm{L}^{\infty}} \leq 1
\end{aligned}\right.
$$

Here $S^{\prime}(u)^{*}$ denotes the adjoint of $S^{\prime}(u)$ with respect to $\mathrm{L}^{2}(D)$, and $j: \mathcal{X} \rightarrow \mathcal{X}^{*}$ is the (linear) duality mapping, i.e., $j(u)=\partial\left(\frac{1}{2}\|u\|_{\mathcal{X}}^{2}\right)$. Note that both $S(u)$ and $y^{\delta}$ are in $\mathrm{L}^{2}(D)$, and hence the duality pairing $\left\langle S(u)-y^{\delta}, p\right\rangle_{\mathrm{L}^{1}, \mathrm{~L}^{\infty}}$ coincides with the standard $\mathrm{L}^{2}$-inner product.

Proof. Setting

$$
\begin{aligned}
\mathcal{F}: \mathcal{X} \rightarrow \mathbb{R}, & u \mapsto \frac{\alpha}{2}\left\|u-u_{0}\right\|_{\mathcal{X}}^{2}, \\
\mathcal{G}: \mathrm{L}^{1}(D) \rightarrow \mathbb{R}, & v \mapsto\|v\|_{\mathrm{L}^{1}},
\end{aligned}
$$


we have that

$$
\mathcal{J}_{\alpha}(u)=\mathcal{F}(u)+\mathcal{G}\left(S(u)-y^{\delta}\right)
$$

Since the operator $S$ is twice Fréchet differentiable ((A2), which implies strict differentiability) and $\mathcal{G}$ is real-valued and convex, the sum and chain rules for the generalized gradient [13, Thms. 2.3.3, 2.3.10] yield that for all $u \in \mathcal{X}$, the functional $\mathcal{J}_{\alpha}$ is Lipschitz continuous near $u$ and the relation

$$
\partial \mathcal{J}_{\alpha}(u)=\mathcal{F}^{\prime}(u)+S^{\prime}(u)^{*} \partial \mathcal{G}\left(S(u)-y^{\delta}\right)
$$

holds. The necessary condition $0 \in \partial \mathcal{J}_{\alpha}\left(u_{\alpha}\right)$ for every local minimizer $u_{\alpha}$ of $\mathcal{J}_{\alpha}$ (see, e.g., [13, Prop. 2.3.2]) and thus implies the existence of a subgradient $p_{\alpha} \in \partial \mathcal{G}\left(S\left(u_{\alpha}\right)-y^{\delta}\right) \subset \mathrm{L}^{\infty}(D)$ such that

$$
0=\alpha j\left(u_{\alpha}-u_{0}\right)+S^{\prime}\left(u_{\alpha}\right)^{*} p_{\alpha}
$$

holds, which is the first relation of (OS). Since $\mathcal{G}$ is convex, the generalized gradient reduces to the convex subdifferential (cf. [13, Prop. 2.2.7]), and by its definition we have the equivalence

$$
p_{\alpha} \in \partial \mathcal{G}\left(S\left(u_{\alpha}\right)-y^{\delta}\right) \Leftrightarrow S\left(u_{\alpha}\right)-y^{\delta} \in \partial \mathcal{G}^{*}\left(p_{\alpha}\right),
$$

where $\mathcal{G}^{*}$ is the Fenchel conjugate of $\mathcal{G}$ (see, e.g., [18, Chap. I.4]), given by the indicator function of the unit ball $B \equiv\left\{p \in \mathrm{L}^{\infty}(D):\|p\|_{\mathrm{L}^{\infty}} \leq 1\right\}$. The subdifferential of $\mathcal{G}^{*}$ coincides with the normal cone to $B$. Consequently, we deduce that $p_{\alpha} \in \partial \mathcal{G}\left(S\left(u_{\alpha}\right)-y^{\delta}\right)$ if and only if

$$
\left\langle S\left(u_{\alpha}\right)-y^{\delta}, p-p_{\alpha}\right\rangle_{\mathrm{L}^{2}} \leq 0
$$

holds for all $p \in \mathrm{L}^{\infty}(D)$ with $\|p\|_{\mathrm{L}^{\infty}} \leq 1$, which is the second relation of (OS).

The following structural information for a solution $u_{\alpha}$ of problem $(\mathcal{P})$ is a direct consequence of (OS) and is of independent interest.

Corollary 2.8. Let $u_{\alpha} \in U_{\text {int }}$ be a minimizer of problem $(\mathcal{P})$ and $p_{\alpha} \in \mathrm{L}^{\infty}(D)$ as given by Theorem 2.7. Then the following relations hold:

$$
\begin{array}{lll}
S\left(u_{\alpha}\right)-y^{\delta}=0 & \text { a.e. on } & \left\{x \in D:\left|p_{\alpha}(x)\right|<1\right\} \\
S\left(u_{\alpha}\right)-y^{\delta} \geq 0 & \text { a.e. on } & \left\{x \in D: p_{\alpha}(x)=1\right\} \\
S\left(u_{\alpha}\right)-y^{\delta} \leq 0 & \text { a.e. on } & \left\{x \in D: p_{\alpha}(x)=-1\right\} .
\end{array}
$$

This can be interpreted as follows: the box constraint on the dual solution $p_{\alpha}$ is active where the data is not attained by the primal solution $u_{\alpha}$. In particular, the dual solution $p_{\alpha}$ acts as a noise indicator.

By using a complementarity function [11,31], we can rewrite the second relation of (OS) as

$$
S\left(u_{\alpha}\right)-y^{\delta}=\max \left(0, S\left(u_{\alpha}\right)-y^{\delta}+c\left(p_{\alpha}-1\right)\right)+\min \left(0, S\left(u_{\alpha}\right)-y^{\delta}+c\left(p_{\alpha}+1\right)\right)
$$

for any $c>0$. This can be further discriminated by pointwise inspection to the following three cases: 
1. $\left(S\left(u_{\alpha}\right)-y^{\delta}\right)(x)>0$ and $p_{\alpha}(x)=1$,

2. $\left(S\left(u_{\alpha}\right)-y^{\delta}\right)(x)<0$ and $p_{\alpha}(x)=-1$,

3. $\left(S\left(u_{\alpha}\right)-y^{\delta}\right)(x)=0$ and $p_{\alpha}(x) \in[-1,1]$.

Consequently, we have the concise relation

$$
p_{\alpha}=\operatorname{sign}\left(S\left(u_{\alpha}\right)-y^{\delta}\right),
$$

from which we obtain a reduced optimality system

$$
\alpha j\left(u_{\alpha}-u_{0}\right)+S^{\prime}\left(u_{\alpha}\right)^{*}\left(\operatorname{sign}\left(S\left(u_{\alpha}\right)-y^{\delta}\right)\right)=0 .
$$

3. Solution by the SSN method. In view of $\left(\mathrm{OS}^{\prime}\right)$ and the lack of smoothness of the sign function, the optimality system (OS) is not differentiable even in a generalized sense, which precludes the application of Newton-type methods. Meanwhile, gradient descent methods are inefficient unless the step lengths are chosen appropriately, which, however, necessarily requires a detailed knowledge of Lipschitz constants. Therefore, we propose to approximate $(\mathcal{P})$ using a local smoothing of the $\mathrm{L}^{1}$ norm. For simplicity, we will consider only $u_{0}=0$ from here on.

3.1. Approximation. To obtain an SSN system, we wish to replace the sign function in $\left(\mathrm{OS}^{\prime}\right)$ by a locally linear smoothing. We therefore consider for $\beta>0$ the smoothed problem

$$
\min _{u \in U}\left\|S(u)-y^{\delta}\right\|_{\mathrm{L}_{\beta}^{1}}+\frac{\alpha}{2}\|u\|_{\mathcal{X}}^{2},
$$

where $\|v\|_{\mathrm{L}_{\beta}^{1}}$ is a Huber-type smoothing of the $\mathrm{L}^{1}$ norm:

$$
\|v\|_{\mathrm{L}_{\beta}^{1}} \equiv \int_{\Omega}|v(x)|_{\beta} d x, \quad|v(x)|_{\beta} \equiv\left\{\begin{array}{cc}
v(x)-\frac{\beta}{2} & \text { if } v(x)>\beta, \\
-v(x)-\frac{\beta}{2} & \text { if } v(x)<-\beta, \\
\frac{1}{2 \beta} v(x)^{2} & \text { if }|v(x)| \leq \beta .
\end{array}\right.
$$

The existence of a minimizer $u_{\beta}$ of $\left(\mathcal{P}_{\beta}\right)$ follows as before. Since the mapping $\psi: \mathbb{R} \rightarrow \mathbb{R}$, $t \mapsto|t|_{\beta}$, is differentiable with a globally Lipschitz continuous derivative $t \mapsto \operatorname{sign}_{\beta}(t)$,

$$
\operatorname{sign}_{\beta}(t) \equiv\left\{\begin{aligned}
1 & \text { if } t>\beta \\
-1 & \text { if } t<-\beta \\
\frac{1}{\beta} t & \text { if }|t| \leq \beta
\end{aligned}\right.
$$

we have that $\psi$ defines a differentiable Nemytskii operator from $\mathrm{L}^{p}(D)$ to $\mathrm{L}^{2}(D)$ for every $p \geq 4$ (see, e.g., [45, Chap. 4.3] and references therein) with pointwise defined derivative $\operatorname{sign}_{\beta}(v) h$. We thus obtain the necessary optimality conditions for a minimizer $u_{\beta} \in U_{\text {int }}$ :

$$
\alpha j\left(u_{\beta}\right)+S^{\prime}\left(u_{\beta}\right)^{*}\left(\operatorname{sign}_{\beta}\left(S\left(u_{\beta}\right)-y^{\delta}\right)\right)=0 .
$$


Remark 3.1. This Huber-type smoothing (which is also used in classical robust estimation [28]) is equivalent to an $\mathrm{L}^{2}$-penalization of the dual variable $p \in \mathrm{L}^{\infty}(D)$ in (OS). To see this, we consider (OS) as the optimality conditions of the primal-dual saddle point problem

$$
\min _{u \in U} \max _{\|p\|_{\mathrm{L}^{\infty}} \leq 1}\left\langle S(u)-y^{\delta}, p\right\rangle_{\mathrm{L}^{2}}+\frac{\alpha}{2}\|u\|_{\mathcal{X}}^{2}
$$

which makes use of the dual representation of the $\mathrm{L}^{1}$-norm. We now introduce for $\beta>0$ the penalized saddle point problem

$$
\min _{u \in U}\left(\max _{\|p\|_{\mathrm{L}^{\infty}} \leq 1}\left\langle S(u)-y^{\delta}, p\right\rangle_{\mathrm{L}^{2}}-\frac{\beta}{2}\|p\|_{\mathrm{L}^{2}}^{2}\right)+\frac{\alpha}{2}\|u\|_{\mathcal{X}}^{2} .
$$

The corresponding optimality conditions for minimizers in $U_{\text {int }}$ are given by

$$
\left\{\begin{array}{l}
S^{\prime}\left(u_{\beta}\right)^{*} p_{\beta}+\alpha j\left(u_{\beta}\right)=0, \\
\left\langle S\left(u_{\beta}\right)-y^{\delta}-\beta p_{\beta}, p-p_{\beta}\right\rangle_{\mathrm{L}^{2}} \leq 0
\end{array}\right.
$$

for all $p \in \mathrm{L}^{\infty}(D)$ with $\|p\|_{\mathrm{L}^{\infty}} \leq 1$. By expressing the variational inequality again using a complementarity function with $c=\beta$, we obtain by pointwise inspection that

$$
p_{\beta}=\operatorname{sign}_{\beta}\left(S\left(u_{\beta}\right)-y^{\delta}\right) .
$$

Inserting this expression into the first relation of (3.1) yields precisely $\left(\mathrm{OS}_{\beta}\right)$.

We next show the convergence of solutions to the approximating problems $\left(\mathcal{P}_{\beta}\right)$ to a solution to problem $(\mathcal{P})$.

Theorem 3.2. As $\beta \rightarrow 0$, the family $\left\{u_{\beta}\right\}_{\beta>0} \subset U$ of minimizers of $\left(\mathcal{P}_{\beta}\right)$ contains a subsequence converging in $\mathcal{X}$ to a minimizer of $(\mathcal{P})$.

Proof. Note that for any $\beta>0$, there holds $|v(x)|_{\beta} \leq|v(x)|$, and consequently

$$
\left\|S\left(u_{\alpha}\right)-y^{\delta}\right\|_{\mathrm{L}_{\beta}^{1}} \leq\left\|S\left(u_{\alpha}\right)-y^{\delta}\right\|_{\mathrm{L}^{1}} .
$$

Now the minimizing property of $u_{\beta}$ implies

$$
\left\|S\left(u_{\beta}\right)-y^{\delta}\right\|_{\mathrm{L}_{\beta}^{1}}+\frac{\alpha}{2}\left\|u_{\beta}\right\|_{\mathcal{X}}^{2} \leq\left\|S\left(u_{\alpha}\right)-y^{\delta}\right\|_{\mathrm{L}_{\beta}^{1}}+\frac{\alpha}{2}\left\|u_{\alpha}\right\|_{\mathcal{X}}^{2},
$$

from which it follows that the family $\left\{u_{\beta}\right\}$ is uniformly bounded in $U$. Therefore, there exists a subsequence, also denoted by $\left\{u_{\beta}\right\}$, and some $u^{*} \in U \subset \mathcal{X}$ such that $u_{\beta} \rightarrow u^{*}$ in $\mathcal{X}$. By the strong continuity of $S$ (cf. (A1)), we have $S\left(u_{\beta}\right) \rightarrow S\left(u^{*}\right)$ in $\mathrm{L}^{2}$, and this convergence is pointwise almost everywhere after possibly passing to a further subsequence [21]. In addition, since $|t|_{\beta} \rightarrow|t|$ as $\beta \rightarrow 0$ for every $t \in \mathbb{R}$, we have that $\left|S\left(u_{\alpha}\right)-y^{\delta}\right|_{\beta}$ converges pointwise to $\left|S\left(u_{\alpha}\right)-y^{\delta}\right|$. Fatou's Lemma then implies

$$
\left\|S\left(u^{*}\right)-y^{\delta}\right\|_{\mathrm{L}^{1}}=\int_{D} \lim _{\beta \rightarrow 0}\left|S\left(u_{\beta}\right)-y^{\delta}\right|_{\beta} d x \leq \liminf _{\beta \rightarrow 0}\left\|S\left(u_{\beta}\right)-y^{\delta}\right\|_{\mathrm{L}_{\beta}^{1}} .
$$

Meanwhile, by virtue of Lebesgue's dominated convergence theorem [21], we deduce

$$
\lim _{\beta \rightarrow 0}\left\|S\left(u_{\alpha}\right)-y^{\delta}\right\|_{\mathrm{L}_{\beta}^{1}}=\left\|S\left(u_{\alpha}\right)-y^{\delta}\right\|_{\mathrm{L}^{1}} .
$$


These three relations together with the weak lower semicontinuity of norms indicate

$$
\left\|S\left(u^{*}\right)-y^{\delta}\right\|_{\mathrm{L}^{1}}+\frac{\alpha}{2}\left\|u^{*}\right\|_{\mathcal{X}}^{2} \leq\left\|S\left(u_{\alpha}\right)-y^{\delta}\right\|_{\mathrm{L}^{1}}+\frac{\alpha}{2}\left\|u_{\alpha}\right\|_{\mathcal{X}}^{2} .
$$

This together with the minimizing property of $u_{\alpha}$ implies that $u^{*}$ is a minimizer of $(\mathcal{P})$.

To conclude the proof, it suffices to show that $\limsup _{\beta \rightarrow 0}\left\|u_{\beta}\right\|_{\mathcal{X}} \leq\left\|u^{*}\right\|_{\mathcal{X}}$ holds. To this end, we assume the contrary, i.e., that there exists a subsequence of $\left\{u_{\beta}\right\}_{\beta>0}$, also denoted by $\left\{u_{\beta}\right\}$, satisfying $u_{\beta} \rightarrow u^{*}$ in $\mathcal{X}$ and $\lim _{\beta \rightarrow 0}\left\|u_{\beta}\right\|_{\mathcal{X}} \equiv c>\left\|u^{*}\right\|_{\mathcal{X}}$. Letting $u_{\alpha}=u^{*}$ and $\beta \rightarrow 0$ in (3.2), we arrive at

$$
\limsup _{\beta \rightarrow 0}\left\|S\left(u_{\beta}\right)-y^{\delta}\right\|_{L_{\beta}^{1}}+\frac{\alpha}{2} c^{2} \leq\left\|S\left(u^{*}\right)-y^{\delta}\right\|_{\mathrm{L}^{1}}+\frac{\alpha}{2}\left\|u^{*}\right\|_{\mathcal{X}}^{2},
$$

i.e., $\lim \sup _{\beta \rightarrow 0}\left\|S\left(u_{\beta}\right)-y^{\delta}\right\|_{\mathrm{L}_{\beta}^{1}}<\left\|S\left(u^{*}\right)-y^{\delta}\right\|_{\mathrm{L}^{1}}$, which is in contradiction with the weak lower semicontinuity in (3.3). This concludes the proof.

3.2. SSN method. To solve the optimality system $\left(\mathrm{OS}_{\beta}\right)$ with a semi-smooth Newton method $[11,23,36,46]$, we consider it as an operator equation $F(u)=0$ for $F: U \subset \mathcal{X} \rightarrow \mathcal{X}^{*}$,

$$
F(u)=\alpha j(u)+S^{\prime}(u)^{*}\left(\operatorname{sign}_{\beta}\left(S(u)-y^{\delta}\right)\right) .
$$

We now argue the Newton differentiability of $F$. We recall that a mapping $F: X \rightarrow Y$ between Banach spaces $X$ and $Y$ is Newton differentiable at $x \in X$ if there exists a neighborhood $N(x)$ and a mapping $G: N(x) \rightarrow L(X, Y)$ with

$$
\lim _{\|h\| \rightarrow 0} \frac{\|F(x+h)-F(x)-G(x+h) h\|_{Y}}{\|h\|_{X}} \rightarrow 0 .
$$

(Note that in contrast to Fréchet differentiability, the linearization is taken in a neighborhood $N(x)$ of $x$.) Any mapping $D_{N} F \in\{G(s): s \in N(x)\}$ is then a Newton derivative of $F$ at $x$.

Since $t \mapsto \operatorname{sign}_{\beta}(t)$ is a globally Lipschitz continuous mapping from $\mathbb{R}$ to $\mathbb{R}$, the corresponding Nemytskii operator $p \mapsto \operatorname{sign}_{\beta}(p)$ is Newton differentiable from $L^{p}$ to $L^{q}$ for any $p>q \geq 1[41,46]$, and a Newton derivative is given pointwise by

$$
\left(D_{N} \operatorname{sign}_{\beta}(p) h\right)(x)= \begin{cases}0 & \text { if }|p(x)|>\beta \\ \frac{1}{\beta} h(x) & \text { if }|p(x)| \leq \beta\end{cases}
$$

This yields Newton differentiability of $\operatorname{sign}_{\beta}$ from $\mathcal{Y} \hookrightarrow \mathrm{L}^{q}(D), q>2$, to $\mathrm{L}^{2}(D)$. By the chain rule and the Fréchet differentiability of $S$, it follows that $P: U \rightarrow \mathrm{L}^{2}(D)$,

$$
P(u)=\operatorname{sign}_{\beta}\left(S(u)-y^{\delta}\right),
$$

is Newton differentiable as well, and a Newton derivative acting on a direction $v \in \mathcal{X}$ is given as

$$
D_{N} P(u) v=\beta^{-1} \chi_{\mathcal{I}}\left(S^{\prime}(u) v\right)
$$


Here, $\chi_{\mathcal{I}}$ is defined pointwise for $x \in D$ by

$$
\chi_{\mathcal{I}}(x)= \begin{cases}1 & \text { if }\left|\left(S(u)-y^{\delta}\right)(x)\right| \leq \beta \\ 0 & \text { else }\end{cases}
$$

For a given $u^{k}$, one Newton step consists in solving for the increment $\delta u \in \mathcal{X}$ in

$$
\alpha j^{\prime}\left(u^{k}\right) \delta u+\left(S^{\prime \prime}\left(u^{k}\right) \delta u\right)^{*} P\left(u^{k}\right)+\beta^{-1} S^{\prime}\left(u^{k}\right)^{*}\left(\chi_{\mathcal{I}^{k}} S^{\prime}\left(u^{k}\right) \delta u\right)=-F\left(u^{k}\right)
$$

and setting $u^{k+1}=u^{k}+\delta u$. Given a way to compute the action of the derivatives $S^{\prime}(u) v$, $S^{\prime}(u)^{*} v$, and $\left[S^{\prime \prime}(u) v\right]^{*} p$ for given $u, p$, and $v$ (given in Appendix A for the model problems), system (3.5) can be solved iteratively, e.g., using a Krylov method.

It remains to show the uniform well-posedness of system (3.5), from which superlinear convergence of the SSN method follows by standard arguments. Since the operator $S$ is nonlinear and the functional is possibly nonconvex, we assume the following condition at a minimizer $u_{\beta}$ : There exists a constant $\gamma>0$ such that

$$
\left\langle S^{\prime \prime}\left(u_{\beta}\right)(h, h), P\left(u_{\beta}\right)\right\rangle_{\mathrm{L}^{2}}+\alpha\|h\|_{\mathcal{X}}^{2} \geq \gamma\|h\|_{\mathcal{X}}^{2}
$$

holds for all $h \in \mathcal{X}$. This is related to standard second-order sufficient optimality conditions in PDE-constrained optimization (see, e.g., [45, Chap. 4.10]). The condition is satisfied for either large $\alpha$ or sparse residual $S\left(u_{\beta}\right)-y^{\delta}$, since

$$
\left\langle S^{\prime \prime}\left(u_{\beta}\right)(h, h), P\left(u_{\beta}\right)\right\rangle_{\mathrm{L}^{2}}+\alpha\|h\|_{\mathcal{X}}^{2} \geq\left(\alpha-C\left\|P\left(u_{\beta}\right)\right\|_{\mathrm{L}^{2}}\right)\|h\|_{\mathcal{X}}^{2}
$$

holds by the a priori estimate on $S^{\prime \prime}(\mathrm{A} 4)$. In the context of parameter identification problems, this is a reasonable assumption, since for a large noise level, $\alpha$ would take a large value, while a small $\alpha$ is chosen only for small noise levels (which, given the impulsive nature of the noise, is equivalent to strong sparsity of the residual). In the latter case, we observe that $P\left(u_{\beta}\right)=\operatorname{sign}_{\beta}\left(S\left(u_{\beta}\right)-y^{\delta}\right)$ can be expected to be small due to the $\mathrm{L}^{2}$ smoothing of $\operatorname{sign}_{\beta}$. (See Remark 3.1 and note that $P\left(u_{\beta}\right)=p_{\beta}$.) Condition (3.6) is thus satisfied if either $\alpha$ or $\beta$ is sufficiently large. However, this property depends on $\beta$, which together with Theorem 3.2 motivates the use of a continuation strategy in $\beta$; see section 3.3. We note that in general it is not possible to check such conditions a priori even for quadratic functionals.

Proposition 3.3. Let $\beta>0$ be given. If condition (3.6) holds, then for each $u \in U$ sufficiently close to a solution $u_{\beta} \in U_{\mathrm{int}}$ of $\left(\mathrm{OS}_{\beta}\right)$, the mapping $D_{N} F: \mathcal{X} \rightarrow \mathcal{X}^{*}$,

$$
D_{N} F(u)=\alpha j^{\prime}(u)+S^{\prime \prime}(u)^{*} P(u)+\beta^{-1} S^{\prime}(u)^{*} \chi_{I} S^{\prime}(u),
$$

is invertible, and there exists a constant $C>0$ independent of $u$ such that

$$
\left\|\left(D_{N} F\right)^{-1}\right\|_{L\left(\mathcal{X}^{*}, \mathcal{X}\right)} \leq C .
$$

Proof. For given $w \in \mathcal{X}^{*}$, we need to find $\delta u \in \mathcal{X}$ satisfying

$$
\left\langle\alpha j^{\prime}(u) \delta u+\left(S^{\prime \prime}(u) \delta u\right)^{*} P(u)+\beta^{-1} S^{\prime}(u)^{*} \chi_{I} S^{\prime}(u) \delta u, v\right\rangle_{\mathcal{X}^{*}, \mathcal{X}}=\langle w, v\rangle_{\mathcal{X}^{*}, \mathcal{X}}
$$


for all $v \in \mathcal{X}$. Letting $v=\delta u$ and observing that $\left\langle j^{\prime}(u) v, v\right\rangle_{\mathcal{X}^{*}, \mathcal{X}}=\|v\|_{\mathcal{X}}^{2}$ (since $\mathcal{X}$ is a Hilbert space), we obtain

$$
\alpha\|\delta u\|_{\mathcal{X}}^{2}+\left\langle S^{\prime \prime}(u)(\delta u, \delta u), P(u)\right\rangle_{\mathrm{L}^{2}}+\beta^{-1}\left\|\chi_{I} S^{\prime}(u) \delta u\right\|_{\mathrm{L}^{2}}^{2}=\langle w, \delta u\rangle_{\mathcal{X}^{*}, \mathcal{X}} .
$$

Now the pointwise contraction property of the min and the max function implies

$$
\begin{aligned}
\left\|P\left(u_{\beta}\right)-P(u)\right\|_{\mathrm{L}^{2}} \leq & \beta^{-1}\left\|S\left(u_{\beta}\right)-S(u)\right\|_{\mathrm{L}^{2}} \\
& +\beta^{-1}\left\|\max \left(0, S\left(u_{\beta}\right)-y^{\delta}-\beta\right)-\max \left(0, S(u)-y^{\delta}-\beta\right)\right\|_{\mathrm{L}^{2}} \\
& +\beta^{-1}\left\|\min \left(0, S\left(u_{\beta}\right)-y^{\delta}+\beta\right)-\min \left(0, S(u)-y^{\delta}+\beta\right)\right\|_{\mathrm{L}^{2}} \\
\leq & 3 \beta^{-1}\left\|S\left(u_{\beta}\right)-S(u)\right\|_{\mathrm{L}^{2}} .
\end{aligned}
$$

Consequently, by the continuity of the mapping $S$, for sufficiently small $\left\|u_{\beta}-u\right\|_{\mathcal{X}}$, we have small $\left\|P\left(u_{\beta}\right)-P(u)\right\|_{L^{2}}$ as well. Thus, by condition (3.6) and the locally uniform boundedness of $S^{\prime \prime}$ (cf. (A4)), there exists an $\varepsilon>0$ such that

$$
\begin{aligned}
\alpha\|\delta u\|_{\mathcal{X}}^{2} & +\left\langle S^{\prime \prime}(u)(\delta u, \delta u), P(u)\right\rangle_{\mathrm{L}^{2}} \\
& =\alpha\|\delta u\|_{\mathcal{X}}^{2}+\left\langle S^{\prime \prime}(u)(\delta u, \delta u), P\left(u_{\beta}\right)\right\rangle_{\mathrm{L}^{2}}+\left\langle S^{\prime \prime}(u)(\delta u, \delta u), P(u)-P\left(u_{\beta}\right)\right\rangle_{\mathrm{L}^{2}} \\
& \geq \gamma\|\delta u\|_{\mathcal{X}}^{2}-C \varepsilon\|\delta u\|_{\mathcal{X}}^{2} \geq \frac{\gamma}{2}\|\delta u\|_{\mathcal{X}}^{2}
\end{aligned}
$$

holds for all $u$ with $\left\|u-u_{\beta}\right\|_{\mathcal{X}} \leq \varepsilon$ if $\varepsilon$ is sufficiently small.

Finally, we deduce by the Cauchy-Schwarz inequality that

$$
\frac{\gamma}{4}\|\delta u\|_{\mathcal{X}}^{2} \leq\|w\|_{\mathcal{X}^{*}}\|\delta u\|_{\mathcal{X}}
$$

This implies the claim.

Newton differentiability and uniform boundedness of Newton derivatives immediately implies superlinear convergence of the SSN method (3.5).

Theorem 3.4. Let $\beta>0$ and condition (3.6) hold. Then the sequence $\left\{u^{k}\right\}$ of iterates in (3.5) converge superlinearly to a solution $u_{\beta} \in U_{\mathrm{int}}$ of $\left(\mathrm{OS}_{\beta}\right)$, provided that $u^{0}$ is sufficiently close to $u_{\beta}$.

Proof. The proof is standard $[11,23,36,46]$ but given here for the sake of completeness. By the definition of the Newton step $u^{k+1}=u^{k}-\left(D_{N} F\left(u^{k}\right)\right)^{-1} F\left(u^{k}\right)$ and $F\left(u_{\beta}\right)=0$, we obtain using Proposition 3.3 that

$$
\begin{aligned}
\left\|u^{k+1}-u_{\beta}\right\|_{\mathcal{X}} & =\left\|\left(D_{N} F\left(u^{k}\right)\right)^{-1}\left[F\left(u^{k}\right)-F\left(u_{\beta}\right)-D_{N} F\left(u^{k}\right)\left(u^{k}-u_{\beta}\right)\right]\right\|_{\mathcal{X}} \\
& \leq C\left\|F\left(u^{k}\right)-F\left(u_{\beta}\right)-D_{N} F\left(u^{k}\right)\left(u^{k}-u_{\beta}\right)\right\|_{\mathcal{X}^{*}} \\
& =C\left\|F\left(u_{\beta}+d^{k}\right)-F\left(u_{\beta}\right)-D_{N} F\left(u_{\beta}+d^{k}\right) d^{k}\right\|_{\mathcal{X}^{*}}
\end{aligned}
$$

with $d^{k}:=u^{k}-u_{\beta} \in \mathcal{X}$. Now the Newton differentiability of $F$ at $u_{\beta}$ implies that

$$
\left\|u^{k+1}-u_{\beta}\right\|_{\mathcal{X}}=o\left(\left\|u^{k}-u_{\beta}\right\|_{\mathcal{X}}\right),
$$


and thus there exists a neighborhood of $u_{\beta}$ such that

$$
\left\|u^{k+1}-u_{\beta}\right\|_{\mathcal{X}}<\frac{1}{2}\left\|u^{k}-u_{\beta}\right\|_{\mathcal{X}}
$$

from which convergence follows by induction. Applying (3.4) then yields the claimed superlinear rate.

3.3. Parameter choice. The regularized formulation $(\mathcal{P})$ of the parameter identification problem $S(u)=y^{\delta}$ requires specifying the regularization parameter $\alpha$, whose correct choice is crucial in practice. Usually, it is determined using a knowledge of the noise level $\delta$ by, e.g., the discrepancy principle (2.1). However, in practice, the noise level $\delta$ may be unknown, rendering such rules inapplicable. To circumvent this issue, we propose a heuristic choice rule based on the following balancing principle [15]: Choose $\alpha$ such that

$$
(\sigma-1)\left\|S\left(u_{\alpha}\right)-y^{\delta}\right\|_{L^{1}}-\frac{\alpha}{2}\left\|u_{\alpha}\right\|_{\mathcal{X}}^{2}=0
$$

is satisfied. The underlying idea of the principle is to balance the data fitting term with the penalty term, and the weight $\sigma>1$ controls the trade-off between them. This weight depends on the relative smoothness of residual and parameter but not on the data realization. The principle does not require knowledge of the noise level and has been successfully applied to linear inverse problems with $\mathrm{L}^{1}$ data fitting [14, 15]. (See also [30] for relevant theoretical analysis.)

We compute a solution $\alpha^{*}$ to the balancing equation (3.8) by the following simple fixed point algorithm proposed in [14]:

$$
\alpha_{k+1}=(\sigma-1) \frac{\left\|S\left(u_{\alpha_{k}}\right)-y^{\delta}\right\|_{L^{1}}}{\frac{1}{2}\left\|u_{\alpha_{k}}\right\|_{\mathcal{X}}^{2}} .
$$

This fixed point algorithm can be derived formally from the model function approach [15]. The convergence can be proved similar to [14] by observing that the proof given there does not depend on the linearity of the forward operator.

Theorem 3.5. If the initial guess $\alpha_{0}$ satisfies $(\sigma-1)\left\|S\left(u_{\alpha_{0}}\right)-y^{\delta}\right\|_{\mathrm{L}^{1}}-\frac{\alpha_{0}}{2}\left\|u_{\alpha_{0}}\right\|_{\mathcal{X}}^{2}<0$, then the sequence $\left\{\alpha_{k}\right\}$ generated by the fixed point algorithm is monotonically decreasing and converges to a solution to (3.8).

Of similar importance is the proper choice of the smoothing parameter $\beta$. If $\beta$ is too large, the desirable structural property of the $\mathrm{L}^{1}$ model will be lost. However, the secondorder condition (3.7) depends on $\beta$ and cannot be expected to hold for arbitrarily small $\beta$. In particular, the convergence radius for the SSN method is likely to shrink as $\beta$ decreases to zero. These considerations motivate the following continuation strategy: Starting with a large $\beta_{0}$ and setting $\beta_{n+1}=q \beta_{n}$ for some $q \in(0,1)$, we compute the solution $u_{\beta_{n}}$ of $\left(\mathrm{OS}_{\beta}\right)$ using the previous solution $u_{\beta_{n}}$ as an initial guess.

A crucial issue is then selecting an appropriate stopping criterion for the continuation. Since we are most interested in the $\mathrm{L}^{1}$ structure of the problem, we base our stopping rule on the following finite termination property of the linear $\mathrm{L}^{1}$ fitting problem [15, Prop. 3.6]: If the active sets coincide for two consecutive iterations of the SSN method, the semismooth 


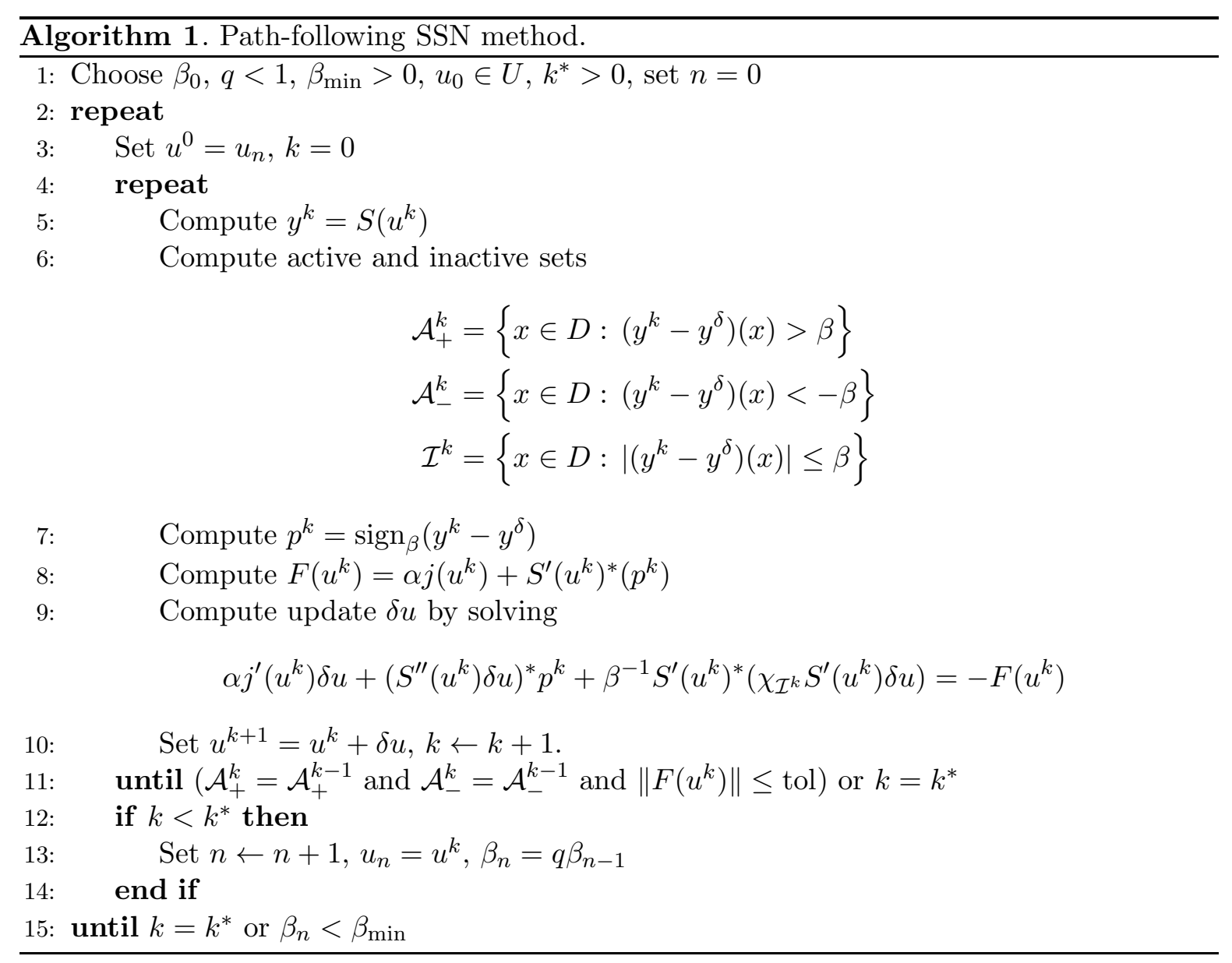

optimality system is solved exactly. In addition, the convergence is usually very fast due to the continuation strategy, and the required number of iterations is independent of the mesh size (this property is well known as mesh independence [25]). Hence, if the active sets (cf. $\mathcal{A}_{+}^{k}$ and $\mathcal{A}_{-}^{k}$ in Algorithm 1) are still changing after a fixed number of iterations, we deduce that the semismoothness of the operator $F(u)$ might be lost and return the last feasible solution $u_{\beta_{n-1}}$ as the desired approximation. In practice, we also check for smallness of the norm of the gradient to take into account the nonlinearity of $S$ and safeguard termination of the algorithm by stopping the continuation if a given very small value $\beta_{\min }$ is reached.

A complete description of this approach, hereafter called the path-following SSN method, is given in Algorithm 1.

4. Numerical examples. We now present some numerical results for several benchmark parameter identification problems with one- and two-dimensional (1d and 2d) elliptic differential equations to illustrate the features of the proposed approach. In each case, the forward operator was discretized using finite elements on a uniform grid (triangular, in the case of two dimensions). We denote by $P_{0}$ the space of piecewise constant functions (on each element), while $P_{1}$ is the space of piecewise linear functions. Unless otherwise stated, the number $N$ of grid points is 1001 in one dimension and $128 \times 128$ in two dimensions. 
We implemented the SSN method as given in Algorithm 1. The iteration was terminated if the active sets did not change and the norm of the gradient fell below $1.00 \times 10^{-6}$, or if 20 iterations were reached. In our experiments, we consider random-valued impulsive noise (cf. section 1.2): Given the true solution $u^{\dagger}$ and the corresponding exact data $y^{\dagger}=S\left(u^{\dagger}\right)$, we set

$$
y^{\delta}= \begin{cases}y^{\dagger} & \text { with probability } 1-r, \\ y^{\dagger}+\left\|y^{\dagger}\right\|_{L^{\infty} \xi} & \text { with probability } r,\end{cases}
$$

where the random variable $\xi$ follows the standard normal distribution and $r \in(0,1)$ is the percentage of corrupted data points. Unless otherwise noted, we take $r=0.3$. The exact noise level $\delta$ is defined by $\delta=\left\|y^{\delta}-y^{\dagger}\right\|_{\mathrm{L}^{1}}$. The Newton system (3.5) is solved iteratively using BiCGstab (with tolerance $1.00 \times 10^{-6}$ and maximum number of iterations 100). The reduction rate $q$ is set to $\frac{1}{2}$.

All timing tests were performed with MATLAB (R2010b) on a single core of a $2.8 \mathrm{GHz}$ workstation with $24 \mathrm{~GB}$ of RAM. The MATLAB codes of our implementation can be downloaded from http://www.uni-graz.at/ ${ }^{\sim}$ clason/codes/11nonlinfit.zip. To keep the presentation concise, all tables are collected in Appendix B.

4.1. Inverse potential problem. This example is concerned with determining the potential $u \in \mathrm{L}^{2}(\Omega)$ in (1.1) from noisy measurements of the state $y \in \mathrm{H}^{1}(\Omega)$ in the domain $\Omega$. The discretized operator $S_{h}$ maps $u_{h} \in U_{h}=P_{0}$ to $y_{h} \in Y_{h}=P_{1}$ which satisfies

$$
\left\langle\nabla y_{h}, \nabla v_{h}\right\rangle_{\mathrm{L}^{2}(\Omega)}+\left\langle u_{h} y_{h}, v_{h}\right\rangle_{\mathrm{L}^{2}(\Omega)}=\left\langle f, v_{h}\right\rangle_{\mathrm{L}^{2}(\Omega)} \quad \text { for all } v_{h} \in Y_{h} .
$$

For the automatic parameter choice using the balancing principle, we have set the weight $\sigma$ to 1.03 and the initial guess $\alpha_{0}$ to 1 .

$1 d$ example. Here, we take $\Omega=[-1,1], f(x)=1$ and

$$
u^{\dagger}(x)=2-|x| \geq 1 \text {. }
$$

A typical realization of noisy data is displayed in Figure 1(a) for $r=0.3$ and Figure 1(b) for $r=0.6$. The fixed point iteration (3.9) converged after 3 (4) iterations for $r=0.3(r=0.6)$, and yielded the values $4.33 \times 10^{-3}\left(9.39 \times 10^{-3}\right)$ for the regularization parameter $\alpha$. The respective reconstructions $u_{\alpha}$, shown in Figures $1(\mathrm{c})$ and $1(\mathrm{~d})$, are nearly indistinguishable from the true solution $u^{\dagger}$. To measure the accuracy of the solution $u_{\alpha}$ quantitatively, we compute the $\mathrm{L}^{2}$-error $e=\left\|u_{\alpha}-u^{\dagger}\right\|_{\mathrm{L}^{2}}$, which is $8.65 \times 10^{-4}$ for $r=0.3$ and $3.32 \times 10^{-3}$ for $r=0.6$. For comparison, we also show the solution by the $\mathrm{L}^{2}$ data fitting problem (solved by a standard Newton method), where the parameter $\alpha$ has been chosen to give the smallest $\mathrm{L}^{2}$-error. We observe that the $\mathrm{L}^{2}$ reconstructions are clearly unacceptable compared to their $\mathrm{L}^{1}$ counterparts, which illustrates the importance of a correct choice of the noise model, and especially the suitability of $\mathrm{L}^{1}$ fitting for impulsive noise.

The performance of the balancing principle is further illustrated in Table 1 (see Appendix B for all tables), where we compare the balancing parameter $\alpha_{\mathrm{b}}$ with the "optimal," samplingbased parameter $\alpha_{\mathrm{o}}$ for different noise levels. This parameter is obtained by sampling each interval $\left[0.1 \alpha_{\mathrm{b}}, \alpha_{\mathrm{b}}\right]$ and $\left[\alpha_{\mathrm{b}}, 10 \alpha_{\mathrm{b}}\right]$ uniformly with 50 parameters and taking as $\alpha_{\mathrm{o}}$ the one 


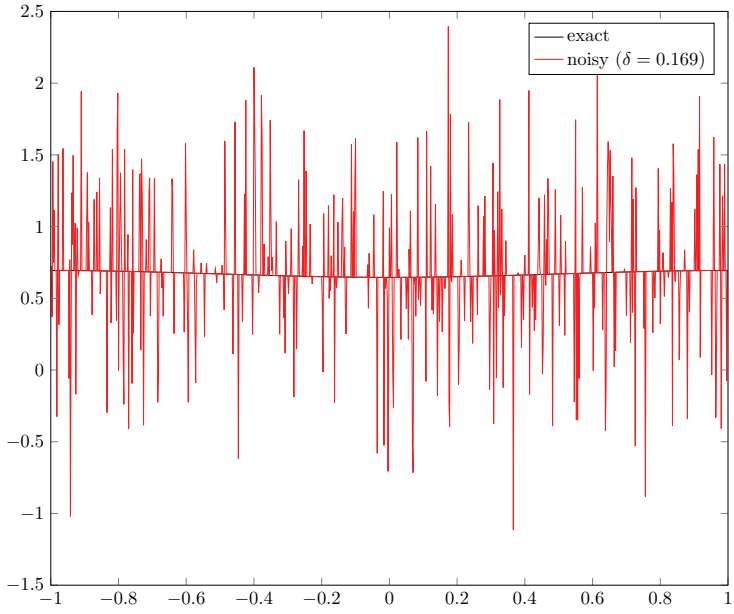

(a) Exact and noisy data $(r=0.3)$

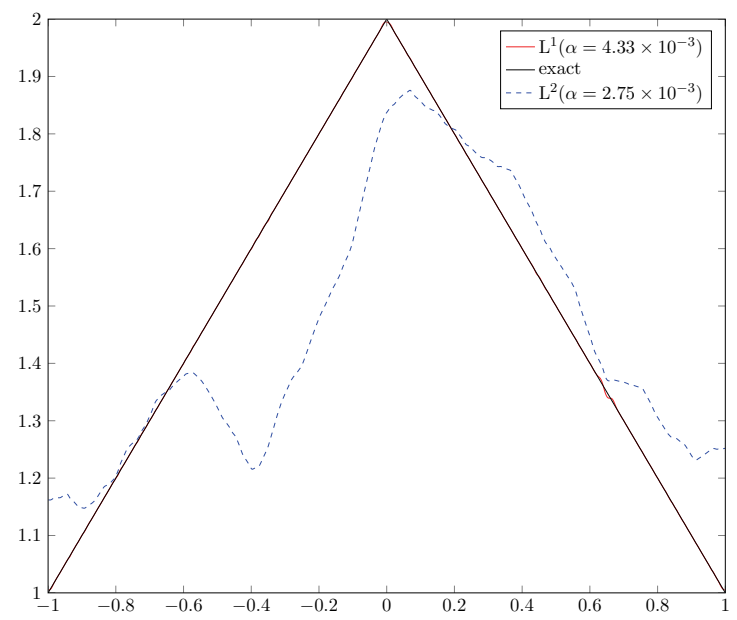

(c) Comparison of $\mathrm{L}^{1}$ and $\mathrm{L}^{2}$ reconstruction

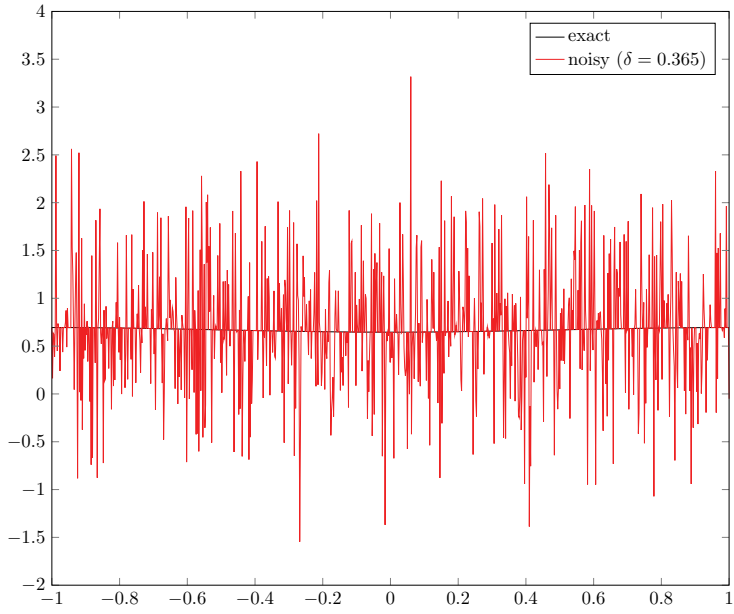

(b) Exact and noisy data $(r=0.6)$

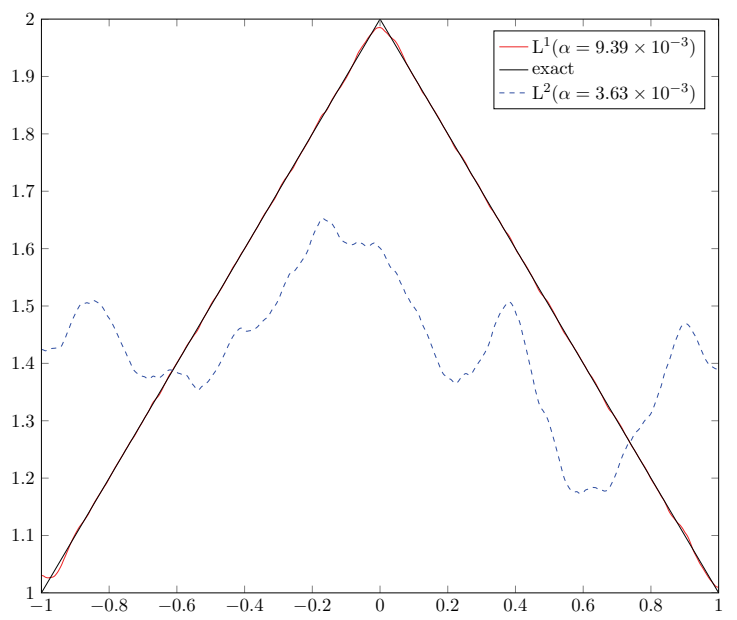

(d) Comparison of $\mathrm{L}^{1}$ and $\mathrm{L}^{2}$ reconstruction

Figure 1. Results for $1 d$ inverse potential problem. Left: $r=0.3$, right: $r=0.6$.

with the smallest $\mathrm{L}^{2}$-error $e_{\mathrm{O}} \equiv\left\|u_{\alpha_{\mathrm{o}}}-u^{\dagger}\right\|_{\mathrm{L}^{2}}$. We observe that both the regularization parameters and the reconstruction errors obtained from the two approaches are comparable. This shows the feasibility of the balancing principle for choosing an appropriate regularization parameter in nonlinear $\mathrm{L}^{1}$ models. Table 1 also illustrates the fundamentally different nature of impulsive noise and $\mathrm{L}^{1}$ fitting compared with Gaussian models, since the $\mathrm{L}^{2}$-error does not depend linearly on the noise level or the percentage $r$ of corrupted data. This can be attributed to the fact that the structural properties of the noise (e.g., clustering of corrupted data points, which is increasingly likely for $r \geq 0.5$ ) is more important than the noise percentage itself.

Next we study the convergence behavior of the path-following SSN method. First, the convergence behavior in the smoothing parameter $\beta$ is illustrated in Table 2 by showing for 
each step in the continuation procedure the value of $\beta$, the required number of SSN iterations, and the $\mathrm{L}^{2}$-error $e$. The required number of SSN iterations is relatively independent of the value of $\beta$ provided it is sufficiently large. Then the semismoothness of the optimality system $\left(\mathrm{OS}_{\beta}\right)$ is gradually lost after the $\beta$ value drops below $1.00 \times 10^{-7}$, and more and more iterations are required for the Krylov method to solve the Newton system (3.5) to the prescribed accuracy. Nonetheless, the reconstruction already represents a very reasonable approximation (in terms of the $\mathrm{L}^{2}$-error $e$ ) at $\beta=1.19 \times 10^{-7}$. Second, we illustrate the superlinear convergence of the SSN method by solving the optimality system (3.1) with fixed $r=0.3, \alpha=4.00 \times 10^{-3}$ and $\beta=1.00 \times 10^{-1}$. Table 3 shows the number of elements that changed between active and inactive sets and the residual norm $\left\|F\left(u^{k}\right)\right\|_{\mathrm{L}^{2}}$ after the $k$ th iteration for several problem sizes $N$. The superlinear convergence as well as the mesh independence can be observed.

Finally, we demonstrate the scalability of the proposed approach. Table 4 summarizes the computing time for one run of the path-following SSN method and for the full fixed point iteration. Since the computing time depends on the $\alpha$ value, we present the results with the final value of $\alpha$ as obtained from the fixed point iteration (3.9). The presented results are the mean and standard deviation over 10 noise realizations. We observe that both the fixed point iteration and the path-following SSN method scale very well with the problem size $N$, which corroborates the mesh independence of the SSN method [25]. We point out that the computational cost of calculating the balancing parameter is only two to three times that of solving the $\mathrm{L}^{1}$ model with one fixed regularization parameter. Therefore, the balancing principle is also computationally inexpensive.

$2 d$ example. Here, we take $\Omega=[-1,1]^{2}, f\left(x_{1}, x_{2}\right)=1$, and

$$
u^{\dagger}\left(x_{1}, x_{2}\right)=1+\cos \left(\pi x_{1}\right) \cos \left(\pi x_{2}\right) \chi_{\left\{\left|\left(x_{1}, x_{2}\right)\right|_{\infty}<1 / 2\right\}} \geq 1 ;
$$

see Figure 2(c). The exact and noisy data (with $r=0.3$ ) are given in Figures 2(a) and 2(b), respectively. The fixed point algorithm (3.9) converged within two iterations to the value $\alpha_{\mathrm{b}}=1.06 \times 10^{-2}$. The solution (with an $\mathrm{L}^{2}$-error $e=5.28 \times 10^{-3}$ ), shown in Figure $2(\mathrm{~d}$ ), accurately captures the shape as well as the magnitude of the potential $u^{\dagger}$ and thus represents a good approximation. The reconstruction by the $\mathrm{L}^{2}$ model is again far from the true solution and thus is not shown here.

4.2. Inverse Robin coefficient problem. This example, meant to illustrate coefficient recovery from boundary data, concerns reconstructing the Robin coefficient $u \in \mathrm{L}^{2}\left(\Gamma_{i}\right)$ in (1.2) from noisy measurements of the Dirichlet trace of $y \in \mathrm{H}^{1}(\Omega)$ on the boundary $\Gamma_{c}$. The discretization $S_{h}$ of the forward operator $S$ thus maps $u_{h} \in U_{h}=P_{0}\left(\Gamma_{i}\right)$ to the restriction of $y_{h} \in Y_{h}=P_{1}$ to the nodes on $\Gamma_{c}$, where $y_{h}$ satisfies

$$
\left\langle\nabla y_{h}, \nabla v_{h}\right\rangle_{\mathrm{L}^{2}(\Omega)}+\left\langle u_{h} y_{h}, v_{h}\right\rangle_{\mathrm{L}^{2}\left(\Gamma_{i}\right)}=\left\langle f, v_{h}\right\rangle_{\mathrm{L}^{2}\left(\Gamma_{c}\right)} \quad \text { for all } v_{h} \in Y_{h} .
$$

Here, we take the domain $\Omega=[0,1]^{2}$, inaccessible boundary $\Gamma_{i}=\left\{\left(x_{1}, x_{2}\right) \in \partial \Omega: x_{1}=1\right\}$, and accessible (contact) boundary $\Gamma_{c}=\partial \Omega \backslash \Gamma_{i}$. Further, we set $f\left(x_{1}, x_{2}\right)=-4+x_{1}$ and

$$
u^{\dagger}\left(x_{2}\right)=1+x_{2} \geq 1 \text {. }
$$

For the automatic parameter choice using the balancing principle, we have set the weight $\sigma$ to 1.03 and the initial guess $\alpha_{0}$ to 1 as before. 


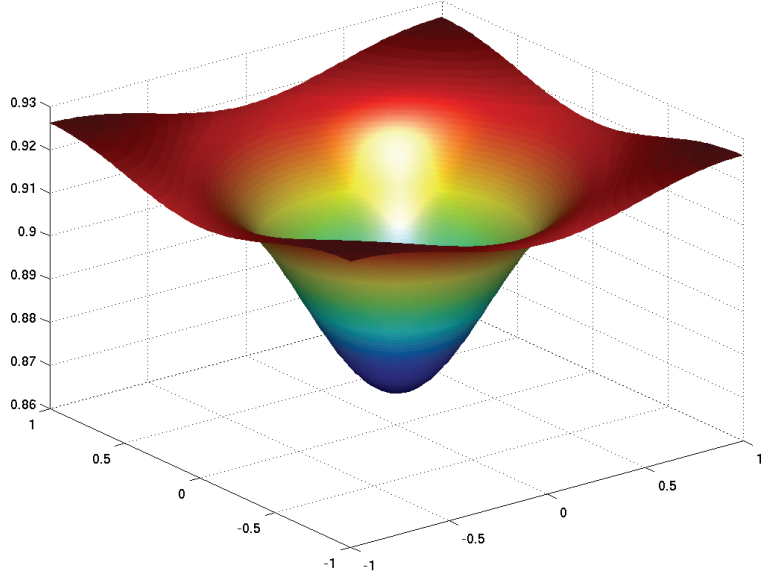

(a) Exact data

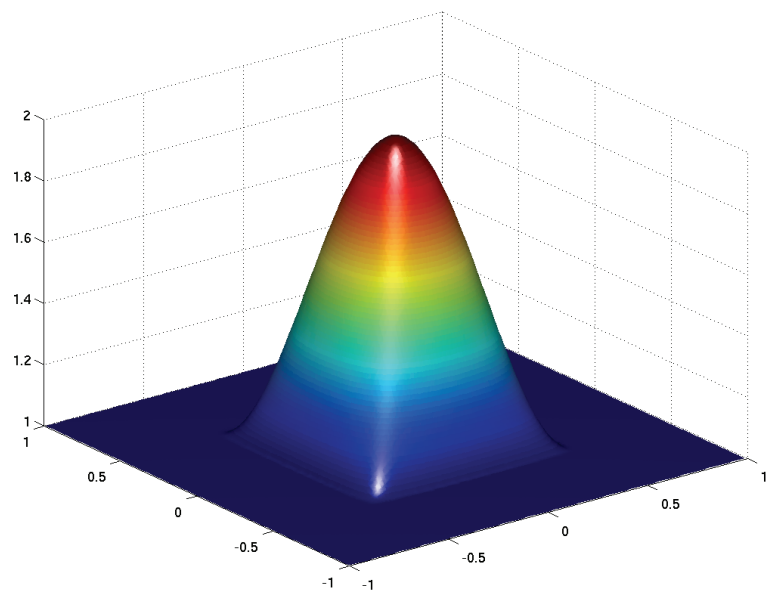

(c) True solution

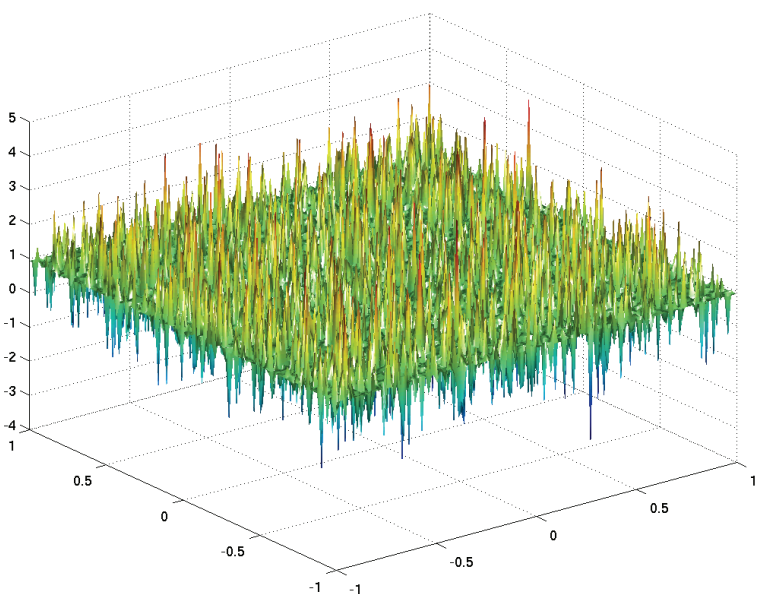

(b) Noisy data $(r=0.3)$

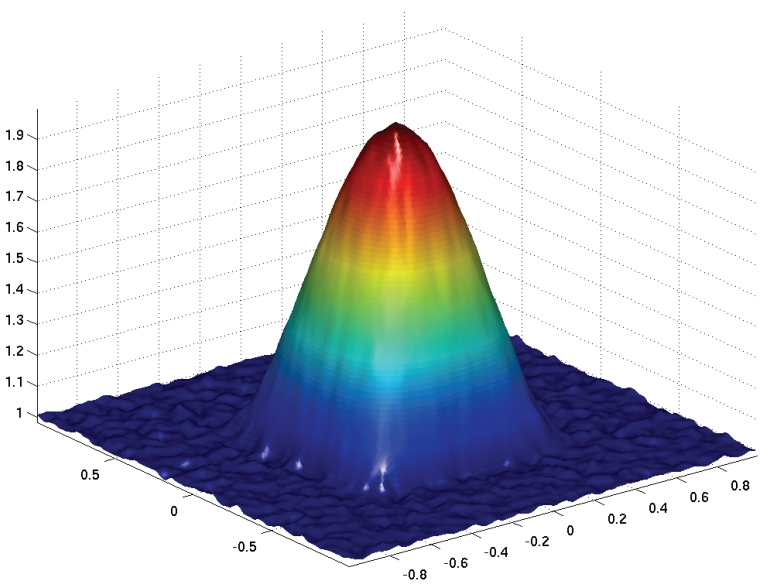

(d) $\mathrm{L}^{1}$ reconstruction $\left(\alpha=1.06 \times 10^{-2}\right)$

Figure 2. Results for the $2 d$ inverse potential problem with $r=0.3\left(\delta=2.24 \times 10^{-1}\right)$.

The noisy data for $r=0.3$ and $r=0.6$ are displayed in Figures 3(a) and 3(b), respectively. The fixed point algorithm (3.9) converged after two iterations in both cases, giving a value $9.77 \times 10^{-2}(r=0.3)$ and $2.12 \times 10^{-1}(r=0.6)$ for the regularization parameter $\alpha$. The corresponding reconstructions $u_{\alpha}$, with respective $\mathrm{L}^{2}$-error $3.13 \times 10^{-3}$ and $1.05 \times 10^{-2}$, are shown in Figures 3(c) and 3(d). Overall, the approximate solutions agree well with the true coefficient, except around the two end points, where the reconstructions suffer from pronounced boundary effect, especially in case of $r=0.6$. Again, the reconstruction by the $\mathrm{L}^{2}$ model (with optimal choice of $\alpha$ ) is not acceptable and thus is not shown. A comparison of the balancing principle with the optimal choice based on sampling is given in Table 5 . The results by these two approaches are very close to each other. From the table, we also observe the nonmonotonicity of the error as a function of $r$, where the reconstruction error $e$ shows a noticeable jump after $r=0.5$. 


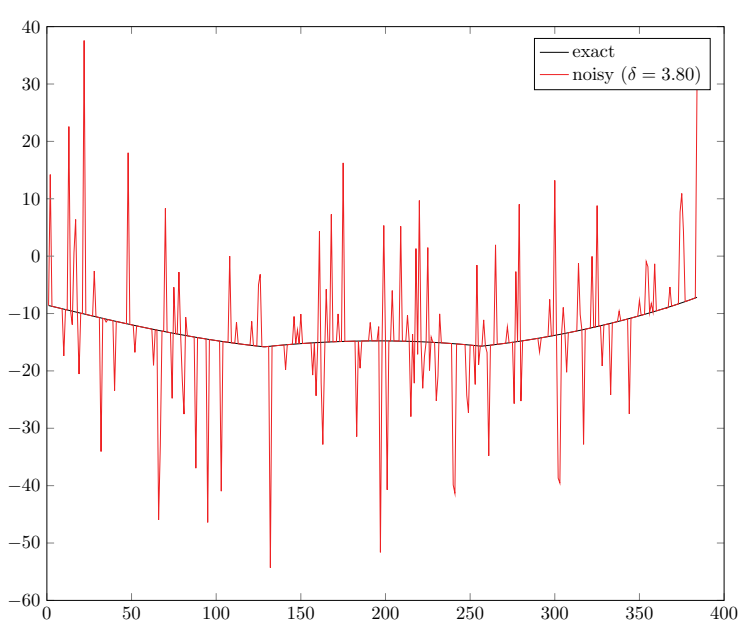

(a) Exact and noisy data $(r=0.3)$

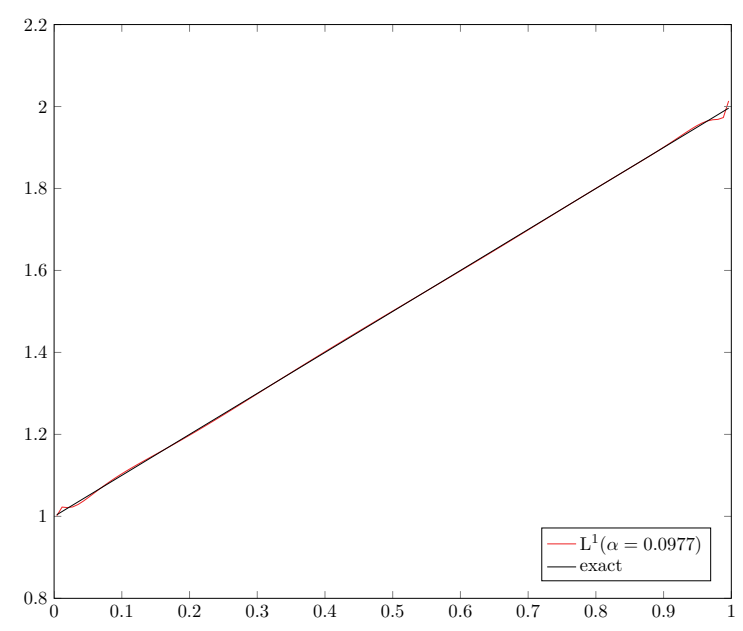

(c) $\mathrm{L}^{1}$ reconstruction

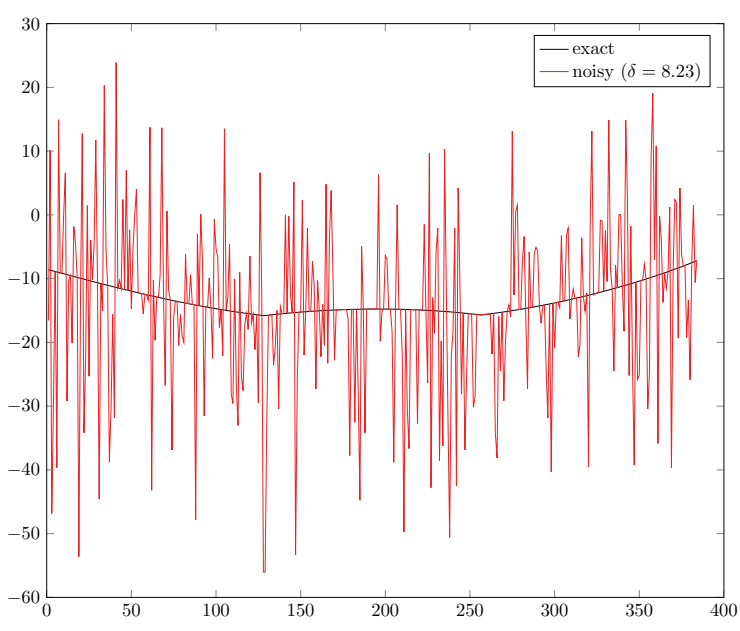

(b) Exact and noisy data $(r=0.6)$

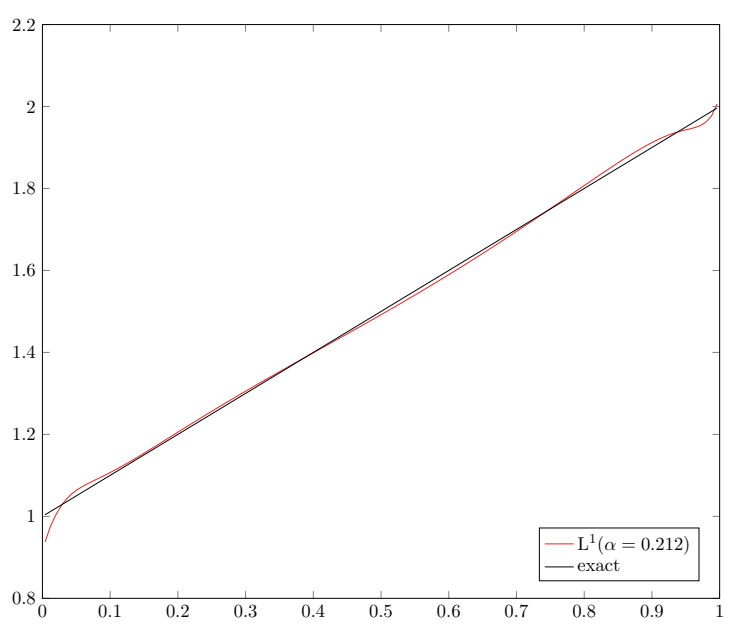

(d) $\mathrm{L}^{1}$ reconstruction

Figure 3. Results for the inverse Robin coefficient problem. Left: $r=0.3$, right: $r=0.6$.

4.3. Inverse diffusion coefficient problem. Finally, we consider the problem of determining the diffusion coefficient $u \in \mathrm{H}^{1}(\Omega)$ in (1.3) from noisy measurements of the solution $y \in \mathrm{H}_{0}^{1}(\Omega)$. Here we take $U_{h}=P_{1}$ and $Y_{h}=P_{1} \cap \mathrm{H}_{0}^{1}(\Omega)$ and consider the discrete operator $S_{h}$ as mapping $u_{h} \in U_{h}$ to $y_{h} \in Y_{h}$ satisfying

$$
\left\langle u_{h} \nabla y_{h}, \nabla v_{h}\right\rangle_{\mathrm{L}^{2}(\Omega)}=\left\langle f, v_{h}\right\rangle_{\mathrm{L}^{2}(\Omega)} \quad \text { for all } v_{h} \in Y_{h}
$$

To accelerate the convergence of the Krylov solver, we precondition the Newton system with the inverse Helmholtz operator $(-\Delta+I)^{-1}$, i.e., the gradient $\alpha(-\Delta u+u)-\nabla y \cdot \nabla p$ is replaced by

$$
\alpha u-(-\Delta+I)^{-1}(\nabla y \cdot \nabla p)
$$




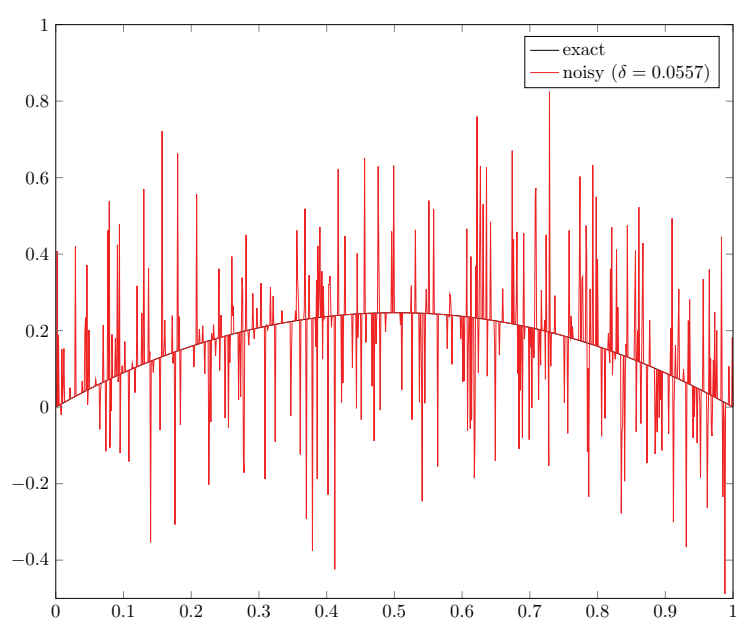

(a) Exact and noisy data $(r=0.3)$

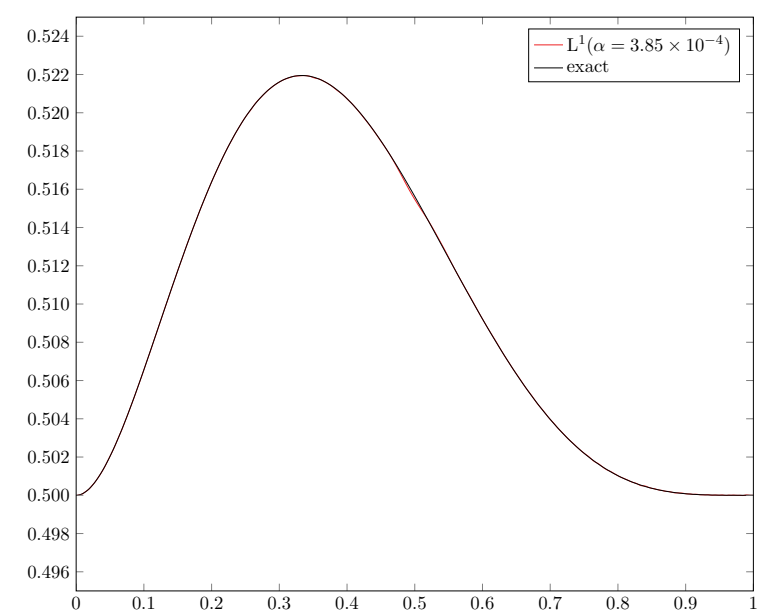

(c) $\mathrm{L}^{1}$ reconstruction

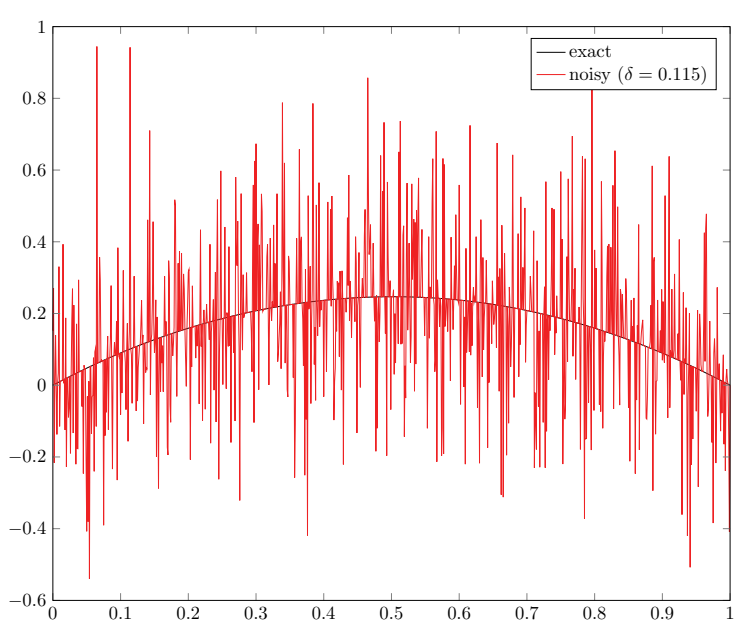

(b) Exact and noisy data $(r=0.6)$

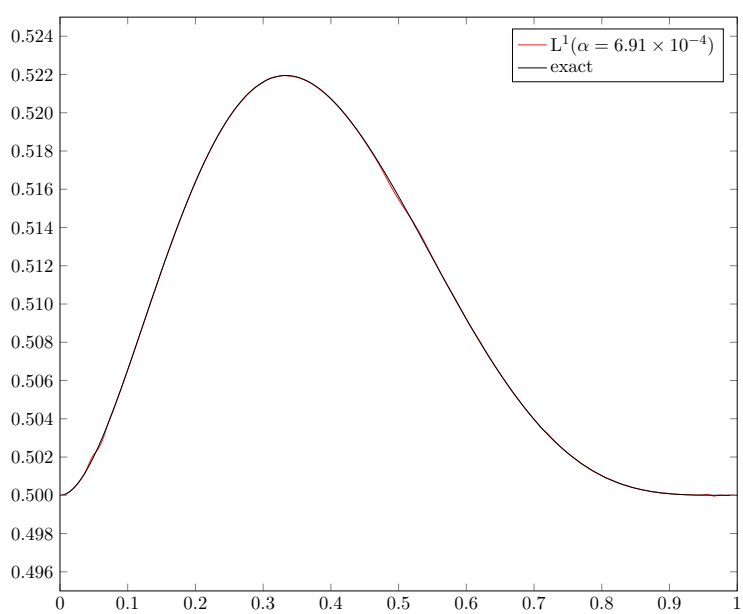

(d) $\mathrm{L}^{1}$ reconstruction

Figure 4. Results for the $1 d$ inverse diffusion coefficient problem. Left: $r=0.3$, right: $r=0.6$.

and similarly the action of the Hessian on $\delta u$ is computed as

$$
\alpha u-(-\Delta+I)^{-1}(\nabla \delta y \cdot \nabla p+\nabla y \cdot \nabla \delta p) .
$$

For the automatic parameter choice using the balancing principle, we have set the weight $\sigma$ to 1.001 and the initial guess $\alpha_{0}$ to 0.1 . As noted, the different weight is chosen according to the stronger smoothness assumption on $u$ ( $\mathrm{H}^{1}$ instead of $\mathrm{L}^{2}$ regularization).

$1 d$ example. Here, we take the domain $\Omega=[0,1]$ and $f(x)=1$. The exact solution $u^{\dagger}$ is given by

$$
u^{\dagger}(x)=\frac{1}{2}+x^{2}(1-x)^{4} \geq \frac{1}{2} .
$$

Noisy data with $r=0.3$ and $r=0.6$ and the reconstructions $\left(\alpha=3.85 \times 10^{-4}, \mathrm{~L}^{2}\right.$-error $2.77 \times 10^{-5}$ and $\alpha=6.90 \times 10^{-4}, \mathrm{~L}^{2}$-error $3.86 \times 10^{-5}$ ) are shown in Figure 4. In both 


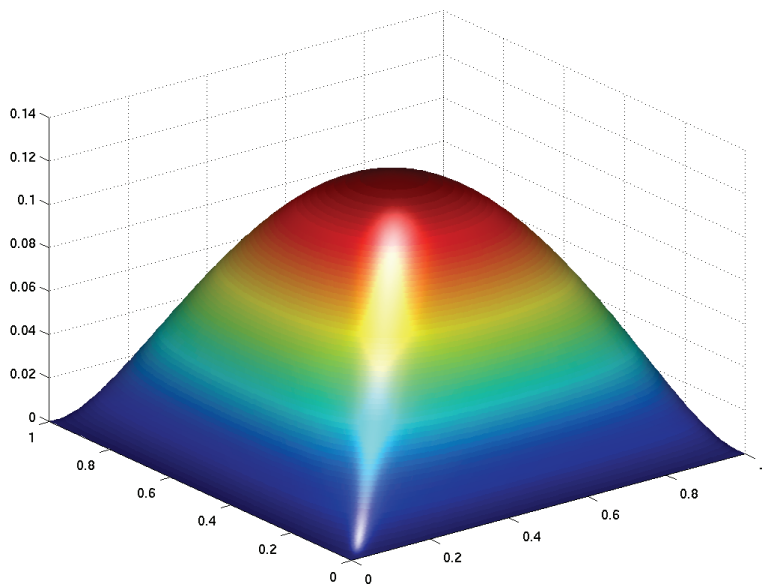

(a) Exact data

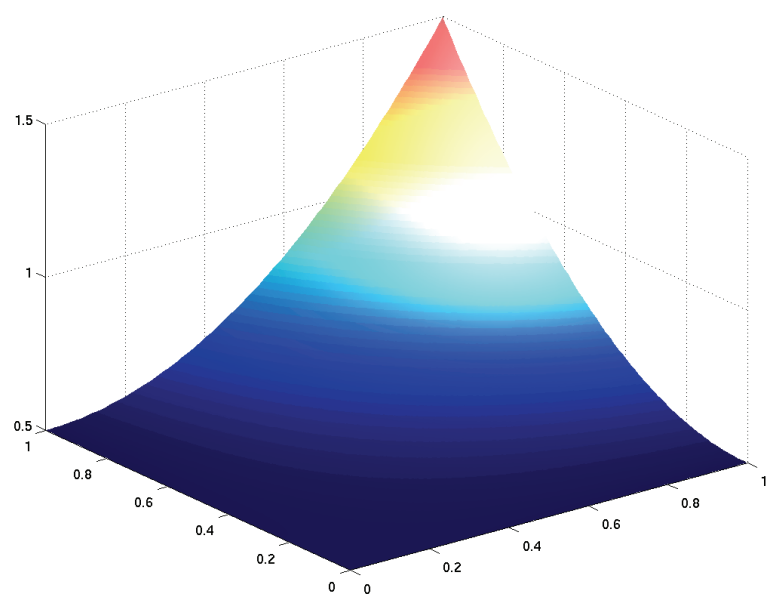

(c) True solution

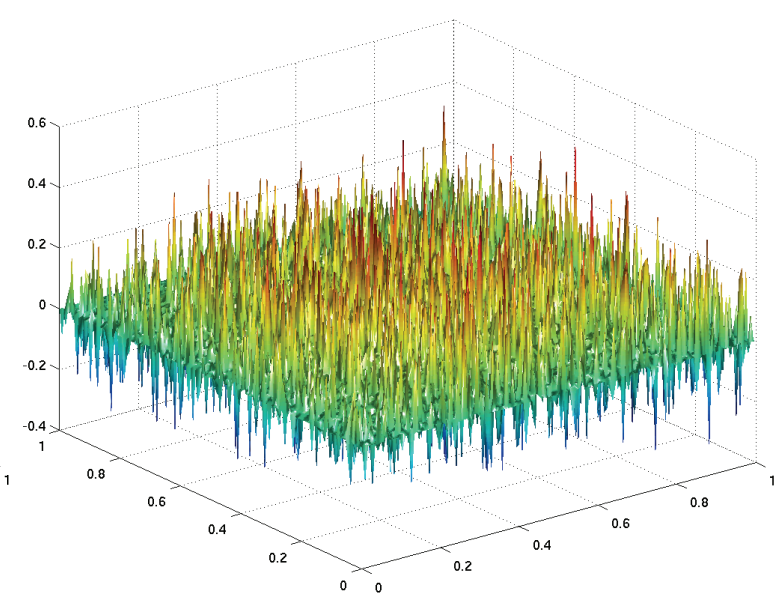

(b) Noisy data $(r=0.3)$

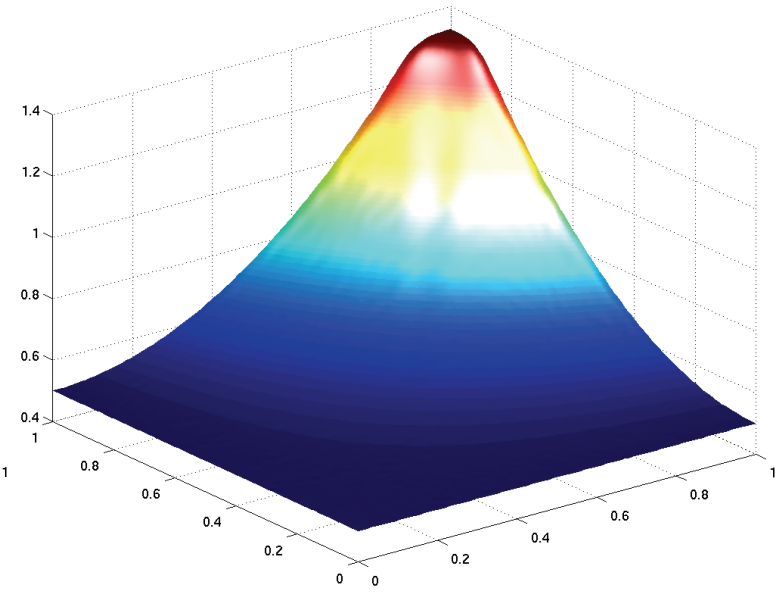

(d) $\mathrm{L}^{1}$ reconstruction $\left(\alpha=5.14 \times 10^{-5}\right)$

Figure 5. Results for the $2 d$ inverse diffusion coefficient problem with $r=0.3\left(\delta=3.06 \times 10^{-2}\right)$.

cases, the fixed point iteration (3.9) converged within two iterations. The convergence of the path-following method and of the SSN method is similar to the inverse potential problem. A comparison of the balancing principle with the optimal choice based on sampling is given in Table 6 . The results by these two approaches are very close to each other. From the table, we also observe the nonmonotonicity of the error as a function of $r$, where the reconstruction error $e$ remains almost constant for $r \leq 0.5$ and then increases quickly. Again the proposed SSN method scales very well with the problem size, as shown in Table 7 .

$2 d$ example. Here, we take $\Omega=[0,1]^{2}, f\left(x_{1}, x_{2}\right)=1$, and

$$
u^{\dagger}\left(x_{1}, x_{2}\right)=\frac{1}{2}+x_{1}^{2} x_{2}^{2} \geq \frac{1}{2}
$$

see Figure $5(\mathrm{c})$. The exact and noisy data $(r=0.3)$ are given in Figures $5(\mathrm{a})$ and $5(\mathrm{~b})$, respectively. The fixed point algorithm converged in seven iterations to the value $\alpha=5.14 \times$ 
$10^{-5}$. The reconstruction, shown in Figure 5(d), agrees well with the true solution (the $\mathrm{L}^{2}$-error being $\left.5.63 \times 10^{-3}\right)$. The less accurate approximation around the corner might be attributed to the fact that the true solution does not satisfy the homogeneous Neumann conditions imposed by the Newton step. Again, we remark that the $\mathrm{L}^{2}$ reconstruction (not presented) is far from the true solution.

5. Conclusion. In this paper we have presented a path-following SSN method for the efficient numerical solution of nonlinear parameter identification problems with impulsive noise. The method is based on a Huber-type smoothing of the $\mathrm{L}^{1}$ fitting functional, and its superlinear convergence is proved and demonstrated numerically. Furthermore, mesh independence of the method can be observed. Several model examples for elliptic differential equations illustrate the efficiency of this approach.

The balancing principle is shown to be an effective parameter choice method, which required little a priori information such as the noise level, while adding only a small amount of computational overhead over the solution of one single minimization problem.

The presented approach can be extended in several directions. As noted in Remark 2.6, including constraints on the solution would be a natural progression. The extension to timedependent problems would be straightforward but poses interesting challenges for efficient implementation. Finally, it would be worthwhile to consider mixed Gaussian and impulsive noise. While such noise is challenging for either $\mathrm{L}^{1}$ or $\mathrm{L}^{2}$ fitting, our robust approximation $\left(\mathcal{P}_{\beta}\right)$ seems to be an appropriate model [28] (cf. also Remark 3.1). Then the continuation $\beta \rightarrow 0$ would have to be replaced by a suitable parameter choice method for determining the optimal stopping value $\beta^{*}>0$. Such an approach might also be applicable to other non-Gaussian models like Laplace and Cauchy noise.

Appendix A. Verification of properties for model problems. For completeness, we collect in this section some results which verify the continuity and differentiability properties (A1) (A4) for our model problems. Throughout, we shall denote by $C$ a generic constant, which is independent of $u \in U$.

A.1. Elliptic potential problem. For this model problem, $S$ maps $u \in \mathcal{X}=\mathrm{L}^{2}(\Omega)$ to the solution $y \in \mathcal{Y}=\mathrm{H}^{1}(\Omega)$ of (1.1), and we take $U=\left\{u \in \mathrm{L}^{\infty}(\Omega): u \geq c\right\}$ for some fixed $c>0$. The verification of properties (A1)-(A4) is analogous to [35]. We therefore only give, for the sake of completeness, the explicit form of the derivatives required for the solution of the Newton system (3.5) using a Krylov subspace method.

For given $u \in \mathrm{L}^{2}(\Omega), F(u)$ is computed by the following steps:

1. Solve for $y \in \mathrm{H}^{1}(\Omega)$ in

$$
\langle\nabla y, \nabla v\rangle_{\mathrm{L}^{2}(\Omega)}+\langle u y, v\rangle_{\mathrm{L}^{2}(\Omega)}=\langle f, v\rangle_{\mathrm{L}^{2}(\Omega)} \quad \text { for all } v \in \mathrm{H}^{1}(\Omega) .
$$

2. Solve for $p \in \mathrm{H}^{1}(\Omega)$ in

$$
\langle\nabla p, \nabla v\rangle_{\mathrm{L}^{2}(\Omega)}+\langle u p, v\rangle_{\mathrm{L}^{2}(\Omega)}=-\left\langle\operatorname{sign}_{\beta}\left(y-y^{\delta}\right), v\right\rangle_{\mathrm{L}^{2}(\Omega)} \quad \text { for all } v \in \mathrm{H}^{1}(\Omega) .
$$

3. Set $F(u)=\alpha u+y p$. 
For given $\delta u \in \mathrm{L}^{2}(\Omega)$, the application of $D_{N} F(u)$ on $\delta u$ is computed by the following:

1. Solve for $\delta y \in \mathrm{H}^{1}(\Omega)$ in

$$
\langle\nabla \delta y, \nabla v\rangle_{\mathrm{L}^{2}(\Omega)}+\langle u \delta y, v\rangle_{\mathrm{L}^{2}(\Omega)}=-\langle y \delta u, v\rangle_{\mathrm{L}^{2}(\Omega)} \quad \text { for all } v \in \mathrm{H}^{1}(\Omega) .
$$

2. Solve for $\delta p \in \mathrm{H}^{1}(\Omega)$ in

$$
\langle\nabla \delta p, \nabla v\rangle_{\mathrm{L}^{2}(\Omega)}+\langle u \delta p, v\rangle_{\mathrm{L}^{2}(\Omega)}=-\left\langle\frac{1}{\beta} \chi_{\mathcal{I}} \delta y+p \delta u, v\right\rangle_{\mathrm{L}^{2}(\Omega)} \quad \text { for all } v \in \mathrm{H}^{1}(\Omega) .
$$

3. Set $D_{N} F(u)=\alpha \delta u+p \delta y+y \delta p$.

A.2. Robin coefficient problem. Here, $S$ maps $u \in \mathcal{X}=\mathrm{L}^{2}\left(\Gamma_{i}\right)$ to $\left.y\right|_{\Gamma_{c}} \in \mathcal{Y}=\mathrm{H}^{\frac{1}{2}}\left(\Gamma_{c}\right)$, where $y$ is the solution to (1.2). Set $U=\left\{u \in \mathrm{L}^{\infty}\left(\Gamma_{i}\right): u \geq c\right\}$ for some fixed $c>0$. We shall denote the mapping of $u \in U$ to the solution $y \in \mathrm{H}^{1}(\Omega)$ of (1.2) by $y(u)$. The following a priori estimate follows directly from the Lax-Milgram theorem.

Lemma A.1. For any $u \in U$, problem (1.2) has a unique solution $y \in \mathrm{H}^{1}(\Omega)$ which satisfies

$$
\|y\|_{\mathrm{H}^{1}(\Omega)} \leq C\|f\|_{\mathrm{H}^{-\frac{1}{2}}\left(\Gamma_{c}\right)} .
$$

Since $f \in \mathrm{H}^{-\frac{1}{2}}\left(\Gamma_{c}\right)$ is fixed, the uniform boundedness of $S$ follows from the continuity of the trace operator. We next address the complete continuity of $S$.

Lemma A.2. Let $\left\{u_{n}\right\} \subset U$ be a sequence converging weakly in $\mathrm{L}^{2}\left(\Gamma_{i}\right)$ to $u^{*} \in U$. Then

$$
S\left(u_{n}\right) \rightarrow S\left(u^{*}\right) \quad \text { in } \mathrm{L}^{2}\left(\Gamma_{c}\right) .
$$

Proof. For $u_{n} \in U$, set $y_{n}=y\left(u_{n}\right) \in \mathrm{H}^{1}(\Omega)$. By the a priori estimate from Lemma A.1, the sequence $\left\{y_{n}\right\}$ is uniformly bounded in $\mathrm{H}^{1}(\Omega)$ and has a convergent subsequence, also denoted by $\left\{y_{n}\right\}$, such that there exists $y^{*} \in \mathrm{H}^{1}(\Omega)$ with

$$
y_{n} \rightarrow y^{*} \text { in } \mathrm{H}^{1}(\Omega) .
$$

The trace theorem and the Sobolev embedding theorem [1] imply

$$
y_{n} \rightarrow y^{*} \text { in } \mathrm{L}^{p}\left(\Gamma_{c}\right)
$$

for any $p<+\infty$. In particular, we will take $p=4$. Then we have

$$
\left|\left\langle u_{n}\left(y_{n}-y^{*}\right), v\right\rangle_{\mathrm{L}^{2}\left(\Gamma_{i}\right)}\right| \leq\left\|u_{n}\right\|_{\mathrm{L}^{2}\left(\Gamma_{i}\right)}\left\|y_{n}-y^{*}\right\|_{\mathrm{L}^{4}\left(\Gamma_{i}\right)}\|v\|_{\mathrm{L}^{4}\left(\Gamma_{i}\right)} \rightarrow 0
$$

by the weak convergence of $\left\{u^{n}\right\}$ in $\mathrm{L}^{2}\left(\Gamma_{i}\right)$ and the strong convergence of $\left\{y_{n}\right\}$ in $\mathrm{L}^{4}\left(\Gamma_{i}\right)$. Therefore, we have

$$
\begin{aligned}
\lim _{n \rightarrow \infty}\left\langle u_{n} y_{n}, v\right\rangle_{\mathrm{L}^{2}\left(\Gamma_{i}\right)} & =\lim _{n \rightarrow \infty}\left(\left\langle u_{n}\left(y_{n}-y^{*}\right), v\right\rangle_{\mathrm{L}^{2}\left(\Gamma_{i}\right)}+\left\langle u_{n} y^{*}, v\right\rangle_{\mathrm{L}^{2}\left(\Gamma_{i}\right)}\right) \\
& =\left\langle u^{*} y^{*}, v\right\rangle_{\mathrm{L}^{2}\left(\Gamma_{i}\right)} .
\end{aligned}
$$

Now passing to the limit in the weak formulation indicates that $y^{*}$ satisfies

$$
\left\langle\nabla y^{*}, \nabla v\right\rangle_{\mathrm{L}^{2}(\Omega)}+\left\langle u^{*} y^{*}, v\right\rangle_{\mathrm{L}^{2}\left(\Gamma_{i}\right)}=\langle f, v\rangle_{\mathrm{L}^{2}\left(\Gamma_{c}\right)} \quad \text { for all } v \in \mathrm{H}^{1}(\Omega)
$$


i.e., $y^{*}=y\left(u^{*}\right)$. Since every subsequence has itself a subsequence converging weakly in $\mathrm{H}^{1}(\Omega)$ to $y\left(u^{*}\right)$, the whole sequence converges weakly. The continuity of $S:\left.u \mapsto y(u)\right|_{\Gamma_{c}}$ then follows from the trace theorem and the Sobolev embedding theorem for $p=2$.

The above two statements imply that property (A1) holds. We next address the remaining properties.

Lemma A.3. The mapping $u \mapsto y(u)$ is twice Fréchet differentiable from $U$ to $\mathrm{H}^{1}(\Omega)$, and for every $u \in U$ and all directions $h_{1}, h_{2} \in \mathrm{L}^{2}\left(\Gamma_{i}\right)$, the derivatives are given by the following:

(i) $y^{\prime}(u) h_{1} \in \mathrm{H}^{1}(\Omega)$ is the solution $z$ of

$$
\langle\nabla z, \nabla v\rangle_{\mathrm{L}^{2}(\Omega)}+\langle u z, v\rangle_{\mathrm{L}^{2}\left(\Gamma_{i}\right)}=-\left\langle h_{1} y(u), v\right\rangle_{\mathrm{L}^{2}\left(\Gamma_{i}\right)}
$$

for all $v \in \mathrm{H}^{1}(\Omega)$, and the following estimate holds:

$$
\left\|y^{\prime}(u) h_{1}\right\|_{\mathrm{H}^{1}(\Omega)} \leq C\left\|h_{1}\right\|_{\mathrm{L}^{2}\left(\Gamma_{i}\right)} .
$$

(ii) $y^{\prime \prime}(u)\left(h_{1}, h_{2}\right) \in \mathrm{H}^{1}(\Omega)$ is the solution $z$ of

$$
\langle\nabla z, \nabla v\rangle_{\mathrm{L}^{2}(\Omega)}+\langle u z, v\rangle_{\mathrm{L}^{2}\left(\Gamma_{i}\right)}=-\left\langle h_{1} y^{\prime}(u) h_{2}, v\right\rangle_{\mathrm{L}^{2}\left(\Gamma_{i}\right)}-\left\langle h_{2} y^{\prime}(u) h_{1}, v\right\rangle_{\mathrm{L}^{2}\left(\Gamma_{i}\right)}
$$

for all $v \in \mathrm{H}^{1}(\Omega)$, and the following estimate holds:

$$
\left\|y^{\prime \prime}(u)\left(h_{1}, h_{2}\right)\right\|_{\mathrm{H}^{1}(\Omega)} \leq C\left\|h_{1}\right\|_{\mathrm{L}^{2}\left(\Gamma_{i}\right)}\left\|h_{2}\right\|_{\mathrm{L}^{2}\left(\Gamma_{i}\right)} .
$$

Proof. The characterization of the derivatives follows from direct calculation. It remains to show boundedness and continuity. By setting $v=y^{\prime}(u) h_{1}$ in the weak formulation, Hölder's inequality, the trace theorem, and the a priori estimate in Lemma A.1, we have

$$
\begin{aligned}
\left\|y^{\prime}(u) h_{1}\right\|_{\mathrm{H}^{1}(\Omega)}^{2} & \leq C\left\|y^{\prime}(u) h_{1}\right\|_{\mathrm{L}^{4}\left(\Gamma_{i}\right)}\left\|h_{1}\right\|_{\mathrm{L}^{2}\left(\Gamma_{i}\right)}\|y(u)\|_{\mathrm{L}^{4}\left(\Gamma_{i}\right)} \\
& \leq C\left\|y^{\prime}(u) h_{1}\right\|_{\mathrm{H}^{1}(\Omega)}\left\|h_{1}\right\|_{\mathrm{L}^{2}\left(\Gamma_{i}\right)}\|y(u)\|_{\mathrm{H}^{1}(\Omega)} \\
& \leq C\left\|y^{\prime}(u) h_{1}\right\|_{\mathrm{H}^{1}(\Omega)}\left\|h_{1}\right\|_{\mathrm{L}^{2}\left(\Gamma_{i}\right)},
\end{aligned}
$$

from which the first estimate follows. Analogously we deduce that

$$
\left\|y\left(u+h_{1}\right)-y(u)\right\|_{\mathrm{H}^{1}(\Omega)} \leq C\left\|h_{1}\right\|_{\mathrm{L}^{2}\left(\Gamma_{i}\right)} .
$$

Next let $w=y\left(u+h_{1}\right)-y(u)-y^{\prime}(u) h_{1}$, which satisfies

$$
\langle\nabla w, \nabla v\rangle_{\mathrm{L}^{2}(\Omega)}+\langle u w, v\rangle_{\mathrm{L}^{2}\left(\Gamma_{i}\right)}=-\left\langle h_{1}\left(y\left(u+h_{1}\right)-y(u)\right), v\right\rangle_{\mathrm{L}^{2}\left(\Gamma_{i}\right)}
$$

for all $v \in \mathrm{H}^{1}(\Omega)$. By repeating the proof of the preceding estimate, we deduce that

$$
\|w\|_{\mathrm{H}^{1}(\Omega)} \leq C\left\|h_{1}\right\|_{\mathrm{L}^{2}\left(\Gamma_{i}\right)}\left\|y\left(u+h_{1}\right)-y(u)\right\|_{\mathrm{H}^{1}(\Omega)},
$$

from which it follows directly that $y^{\prime}(u) h_{1}$ defined above is indeed the Fréchet derivative of $y(u)$ at $u$. By arguing similarly and using the first assertion, the second assertion follows. 
Together with the linearity of the trace operator, we obtain $S^{\prime}(u) h_{1}=\left.y^{\prime}(u) h_{1}\right|_{\Gamma_{c}} \in \mathrm{H}^{\frac{1}{2}}\left(\Gamma_{c}\right)$ and $S^{\prime \prime}(u)\left(h_{1}, h_{2}\right)=\left.y^{\prime \prime}(u)\left(h_{1}, h_{2}\right)\right|_{\Gamma_{c}} \in \mathrm{H}^{\frac{1}{2}}\left(\Gamma_{c}\right)$ and thus property (A2). Finally, properties (A3) and (A4) follow directly from the estimates in Lemma A.3 and the trace theorem [1].

We again give the necessary steps in a Krylov subspace method for the solution to (3.5). For given $u \in \mathrm{L}^{2}\left(\Gamma_{i}\right), F(u)$ is computed by the following steps:

1. Solve for $y \in \mathrm{H}^{1}(\Omega)$ in

$$
\langle\nabla y, \nabla v\rangle_{\mathrm{L}^{2}(\Omega)}+\langle u y, v\rangle_{\mathrm{L}^{2}\left(\Gamma_{i}\right)}=\langle f, v\rangle_{\mathrm{L}^{2}\left(\Gamma_{c}\right)} \quad \text { for all } v \in \mathrm{H}^{1}(\Omega) .
$$

2. Solve for $p \in \mathrm{H}^{1}(\Omega)$ in

$$
\langle\nabla p, \nabla v\rangle_{\mathrm{L}^{2}(\Omega)}+\langle u p, v\rangle_{\mathrm{L}^{2}\left(\Gamma_{i}\right)}=-\left\langle\operatorname{sign}_{\beta}\left(\left.y\right|_{\Gamma_{c}}-y^{\delta}\right), v\right\rangle_{\mathrm{L}^{2}\left(\Gamma_{c}\right)} \quad \text { for all } v \in \mathrm{H}^{1}(\Omega) .
$$

3. Set $F(u)=\alpha u+\left.\left.y\right|_{\Gamma_{i}} p\right|_{\Gamma_{i}}$.

For given $\delta u \in \mathrm{L}^{2}\left(\Gamma_{i}\right)$, the application of $D_{N} F(u)$ on $\delta u$ is computed by the following:

1. Solve for $\delta y \in \mathrm{H}^{1}(\Omega)$ in

$$
\langle\nabla \delta y, \nabla v\rangle_{\mathrm{L}^{2}(\Omega)}+\langle u \delta y, v\rangle_{\mathrm{L}^{2}\left(\Gamma_{i}\right)}=-\langle y \delta u, v\rangle_{\mathrm{L}^{2}\left(\Gamma_{i}\right)} \quad \text { for all } v \in \mathrm{H}^{1}(\Omega) .
$$

2. Solve for $\delta p \in \mathrm{H}^{1}(\Omega)$ in

$$
\langle\nabla \delta p, \nabla v\rangle_{\mathrm{L}^{2}(\Omega)}+\langle u p, v\rangle_{\mathrm{L}^{2}\left(\Gamma_{i}\right)}=-\left\langle\frac{1}{\beta} \chi_{\mathcal{I}}\left(\left.\delta y\right|_{\Gamma_{c}}\right), v\right\rangle_{\mathrm{L}^{2}\left(\Gamma_{c}\right)}-\langle p \delta u, v\rangle_{\mathrm{L}^{2}\left(\Gamma_{i}\right)} \text { for all } v \in \mathrm{H}^{1}(\Omega) .
$$

3. Set $D_{N} F(u)=\alpha \delta u+\left.\left.p\right|_{\Gamma_{i}}(\delta y)\right|_{\Gamma_{i}}+\left.\left.y\right|_{\Gamma_{i}}(\delta p)\right|_{\Gamma_{i}}$.

A.3. Diffusion coefficient problem. In this model problem, the operator $S$ maps $u \in$ $\mathcal{X}=\mathrm{H}^{1}(\Omega)$ to the solution $y \in \mathcal{Y}=\mathrm{W}_{0}^{1, q}(\Omega)$, for some $q>2$, of (1.3), and the admissible set is $U=\left\{u \in \mathrm{H}^{1}(\Omega): \lambda \leq u \leq \lambda^{-1}\right\}$ for some fixed $\lambda \in(0,1)$. The following estimate is an immediate consequence of Theorem 1 in [38], where $Q>2$ is a constant depending only on $\lambda$ and $\Omega$. We shall assume $f \in \mathrm{L}^{q}(\Omega)$ for some $q>Q$.

Lemma A.4. There exists a number $Q>2$ depending only on $\lambda$ and $\Omega$ such that for any $u \in U$ and $q \in(2, Q)$, problem (1.3) has a unique solution $y \in \mathrm{W}_{0}^{1, q}(\Omega)$ which satisfies

$$
\|y\|_{\mathrm{W}^{1, q}(\Omega)} \leq C\|f\|_{\mathrm{L}^{q}(\Omega)} .
$$

From this, the uniform boundedness of $S$ follows since $f \in \mathrm{L}^{q}(\Omega)$ is fixed. We next address the complete continuity of $S$.

Lemma A.5. Let $\left\{u_{n}\right\} \subset U$ be a sequence converging weakly in $\mathrm{H}^{1}(\Omega)$ to $u^{*} \in U$. Then

$$
S\left(u_{n}\right) \rightarrow S\left(u^{*}\right) \quad \text { in } \mathrm{L}^{2}(\Omega)
$$

Proof. For $u_{n} \in U$, set $y_{n}=S\left(u_{n}\right) \in \mathrm{W}_{0}^{1, q}(\Omega)$. By the a priori estimate from Lemma A.4, the sequence $\left\{y_{n}\right\}$ is uniformly bounded in $\mathrm{W}^{1, q}(\Omega)$ and has a convergent subsequence also denoted by $\left\{y_{n}\right\}$ such that there exists $y^{*} \in \mathrm{W}_{0}^{1, q}(\Omega)$ with

$$
y_{n} \rightarrow y^{*} \text { in } \mathrm{W}^{1, q}(\Omega) .
$$


The Rellich-Kondrachov embedding theorem [1, Thm. 6.3] implies

$$
u_{n} \rightarrow u^{*} \text { in } \mathrm{L}^{p}(\Omega)
$$

for any $p<+\infty$. In particular, we will take $p$ such that $\frac{1}{2}+\frac{1}{p}+\frac{1}{q}=1$. Then we have

$$
\left|\left\langle\left(u_{n}-u^{*}\right) \nabla y_{n}, \nabla v\right\rangle_{\mathrm{L}^{2}(\Omega)}\right| \leq\left\|u_{n}-u^{*}\right\|_{\mathrm{L}^{p}(\Omega)}\left\|\nabla y_{n}\right\|_{\mathrm{L}^{q}(\Omega)}\|\nabla v\|_{\mathrm{L}^{2}(\Omega)} \rightarrow 0
$$

by the weak convergence of $\left\{y_{n}\right\}$ in $\mathrm{W}^{1, q}(\Omega)$ and the strong convergence of $\left\{u_{n}\right\}$ in $\mathrm{L}^{p}(\Omega)$. Therefore, we have

$$
\begin{aligned}
\lim _{n \rightarrow \infty}\left\langle u_{n} \nabla y_{n}, \nabla v\right\rangle_{\mathrm{L}^{2}(\Omega)} & =\lim _{n \rightarrow \infty}\left(\left\langle\left(u_{n}-u^{*}\right) \nabla y_{n}, \nabla v\right\rangle_{\mathrm{L}^{2}(\Omega)}+\left\langle u^{*} \nabla y_{n}, \nabla v\right\rangle_{\mathrm{L}^{2}(\Omega)}\right) \\
& =\left\langle u^{*} \nabla y^{*}, \nabla v\right\rangle_{\mathrm{L}^{2}(\Omega)} .
\end{aligned}
$$

Now passing to the limit in the weak formulation indicates that $y^{*}$ satisfies

$$
\left\langle u^{*} \nabla y^{*}, \nabla v\right\rangle_{\mathrm{L}^{2}(\Omega)}=\langle f, v\rangle_{\mathrm{L}^{2}(\Omega)} \quad \text { for all } v \in \mathrm{H}_{0}^{1}(\Omega),
$$

i.e., $y^{*}=S\left(u^{*}\right)$. Since every subsequence has itself a subsequence converging weakly in $\mathrm{W}^{1, q}(\Omega)$ to $S\left(u^{*}\right)$, the whole sequence converges weakly. Applying again the Rellich-Kondrachov embedding theorem [1] for $p=2$ completes the proof of the lemma.

The above two statements imply that property (A1) holds. The next statement yields the remaining properties (A2), (A3), and (A4).

Lemma A.6. The operator $S: U \rightarrow \mathrm{W}_{0}^{1, q}(\Omega)$ is twice Fréchet differentiable, and for every $u \in U$ and all admissible directions $h_{1}, h_{2} \in \mathrm{H}^{1}(\Omega)$, the derivatives are given by the following:

(i) $S^{\prime}(u) h_{1} \in \mathrm{W}_{0}^{1, q}(\Omega)$ is the solution $z$ of

$$
\langle u \nabla z, \nabla v\rangle_{\mathrm{L}^{2}(\Omega)}=-\left\langle h_{1} \nabla S(u), \nabla v\right\rangle_{\mathrm{L}^{2}(\Omega)} \quad \text { for all } v \in \mathrm{H}_{0}^{1}(\Omega),
$$

and the following estimate holds:

$$
\left\|S^{\prime}(u) h_{1}\right\|_{\mathrm{W}^{1, q}(\Omega)} \leq C\left\|h_{1}\right\|_{\mathrm{H}^{1}(\Omega)} .
$$

(ii) $S^{\prime \prime}(u)\left(h_{1}, h_{2}\right) \in \mathrm{W}_{0}^{1, q}(\Omega)$ is the solution $z$ of

$$
\langle u \nabla z, \nabla v\rangle_{\mathrm{L}^{2}(\Omega)}=-\left\langle h_{1} \nabla S^{\prime}(u) h_{2}+h_{2} \nabla S^{\prime}(u) h_{1}, \nabla v\right\rangle_{\mathrm{L}^{2}(\Omega)} \quad \text { for all } v \in \mathrm{H}_{0}^{1}(\Omega),
$$

and the following estimate holds:

$$
\left\|S^{\prime \prime}(u)\left(h_{1}, h_{2}\right)\right\|_{\mathrm{W}^{1, q}(\Omega)} \leq C\left\|h_{1}\right\|_{\mathrm{H}^{1}(\Omega)}\left\|h_{2}\right\|_{\mathrm{H}^{1}(\Omega)} .
$$

Proof. Again, the characterization of the derivatives are obtained by direct calculation. Set $y=S(u) \in \mathrm{W}_{0}^{1, q}(\Omega)$. By Lemma A.4 and Hölder's inequality, we get

$$
\begin{aligned}
\left\|S^{\prime}(u) h_{1}\right\|_{\mathrm{W}^{1, q}(\Omega)} & \leq C\left\|h_{1} \nabla y\right\|_{\mathrm{L}^{q}(\Omega)} \leq\left\|h_{1}\right\|_{\mathrm{L}^{p}(\Omega)}\|\nabla y\|_{\mathrm{L}^{q^{\prime}}(\Omega)} \\
& \leq C\left\|h_{1}\right\|_{\mathrm{H}^{1}(\Omega)}\|\nabla y\|_{\mathrm{L}^{q^{\prime}}(\Omega)} \leq C\left\|h_{1}\right\|_{\mathrm{H}^{1}(\Omega)}
\end{aligned}
$$


with $q^{\prime} \in(q, Q)$ and $\frac{1}{q}=\frac{1}{p}+\frac{1}{q^{\prime}}$, where we have used the Sobolev embedding theorem and the estimate in Lemma A.4. Analogously, we deduce that

$$
\left\|S\left(u+h_{1}\right)-S(u)\right\|_{\mathrm{W}^{1, \tilde{q}}(\Omega)} \leq C\left\|h_{1}\right\|_{\mathrm{H}^{1}(\Omega)},
$$

where the exponent $\tilde{q}$ satisfies $\tilde{q} \in(q, Q)$. Next let $w=S\left(u+h_{1}\right)-S(u)-S^{\prime}(u) h_{1}$, which satisfies

$$
\langle u \nabla w, \nabla v\rangle_{\mathrm{L}^{2}(\Omega)}=-\left\langle h_{1} \nabla\left(S\left(u+h_{1}\right)-S(u)\right), \nabla v\right\rangle_{\mathrm{L}^{2}(\Omega)} \quad \text { for all } v \in \mathrm{H}_{0}^{1}(\Omega) .
$$

Repeating the proof of the preceding estimate, we derive

$$
\|w\|_{W^{1, p}(\Omega)} \leq C\left\|h_{1}\right\|_{\mathrm{H}^{1}(\Omega)}\left\|S\left(u+h_{1}\right)-S(u)\right\|_{W^{1, \tilde{q}}(\Omega)} \cdot
$$

Combining these estimates yields the first assertion, i.e., $S^{\prime}(u) h_{1}$ defined above is indeed the Fréchet derivative of the forward operator $S(u): \mathrm{H}^{1}(\Omega) \rightarrow W_{0}^{1, p}(\Omega)$, and it satisfies the desired estimate. Similarly, the second assertion follows from Lemma A.4 and the first assertion.

We finally address the steps required in a Krylov subspace method for the solution to (3.5). For given $u \in \mathrm{H}^{1}(\Omega), F(u)$ is computed by the following steps:

1. Solve for $y \in \mathrm{H}_{0}^{1}(\Omega)$ in

$$
\langle u \nabla y, \nabla v\rangle_{\mathrm{L}^{2}(\Omega)}=\langle f, v\rangle_{\mathrm{L}^{2}(\Omega)} \quad \text { for all } v \in \mathrm{H}_{0}^{1}(\Omega)
$$

2. Solve for $p \in \mathrm{H}_{0}^{1}(\Omega)$ in

$$
\langle u \nabla p, \nabla v\rangle_{\mathrm{L}^{2}(\Omega)}=\left\langle\operatorname{sign}_{\beta}\left(y-y^{\delta}\right), v\right\rangle_{\mathrm{L}^{2}(\Omega)} \quad \text { for all } v \in \mathrm{H}_{0}^{1}(\Omega) .
$$

3. Set $F(u)=\alpha(-\Delta u+u)-\nabla y \cdot \nabla p$.

For given $\delta u \in \mathrm{H}^{1}(\Omega)$, the application of $D_{N} F(u)$ on $\delta u$ is computed by the following:

1. Solve for $\delta y \in \mathrm{H}_{0}^{1}(\Omega)$ in

$$
\langle u \nabla \delta y, \nabla v\rangle_{\mathrm{L}^{2}(\Omega)}=\langle\delta u \nabla y, \nabla v\rangle_{\mathrm{L}^{2}(\Omega)} \quad \text { for all } v \in \mathrm{H}_{0}^{1}(\Omega) .
$$

2. Solve for $\delta p \in \mathrm{H}_{0}^{1}(\Omega)$ in

$$
\langle u \nabla \delta p, \nabla v\rangle_{\mathrm{L}^{2}(\Omega)}=-\left\langle\frac{1}{\beta} \chi_{\mathcal{I}} \delta y, v\right\rangle_{\mathrm{L}^{2}(\Omega)}+\langle\delta u \nabla p, \nabla v\rangle_{\mathrm{L}^{2}(\Omega)} \quad \text { for all } v \in \mathrm{H}_{0}^{1}(\Omega) .
$$

3. Set $D_{N} F(u)=\alpha(-\Delta \delta u+\delta u)-\nabla \delta y \cdot \nabla p-\nabla y \cdot \nabla \delta p$. 


\section{Appendix B. Tables.}

Table 1

Comparison of the balancing principle $\left(\alpha_{\mathrm{b}}, e_{\mathrm{b}}\right)$ with the sampling-based optimal choice $\left(\alpha_{\mathrm{o}}, e_{\mathrm{o}}\right)$ for the $1 d$ inverse potential problem.

\begin{tabular}{cccccc}
\hline$r$ & $\delta$ & $\alpha_{\mathrm{o}}$ & $\alpha_{\mathrm{b}}$ & $e_{\mathrm{o}}$ & $e_{\mathrm{b}}$ \\
\hline 0.1 & $6.46 \mathrm{e}-2$ & $4.65 \mathrm{e}-3$ & $1.66 \mathrm{e}-3$ & $2.64 \mathrm{e}-4$ & $5.90 \mathrm{e}-4$ \\
0.2 & $1.13 \mathrm{e}-1$ & $5.53 \mathrm{e}-3$ & $2.91 \mathrm{e}-3$ & $3.49 \mathrm{e}-4$ & $4.11 \mathrm{e}-4$ \\
0.3 & $1.68 \mathrm{e}-1$ & $4.17 \mathrm{e}-3$ & $4.32 \mathrm{e}-3$ & $4.60 \mathrm{e}-4$ & $5.06 \mathrm{e}-4$ \\
0.4 & $2.26 \mathrm{e}-1$ & $3.82 \mathrm{e}-3$ & $5.81 \mathrm{e}-3$ & $6.26 \mathrm{e}-4$ & $8.97 \mathrm{e}-4$ \\
0.5 & $2.94 \mathrm{e}-1$ & $3.34 \mathrm{e}-3$ & $7.56 \mathrm{e}-3$ & $3.54 \mathrm{e}-3$ & $5.40 \mathrm{e}-3$ \\
0.6 & $3.19 \mathrm{e}-1$ & $6.72 \mathrm{e}-3$ & $8.20 \mathrm{e}-3$ & $1.31 \mathrm{e}-2$ & $2.05 \mathrm{e}-2$ \\
0.7 & $3.86 \mathrm{e}-1$ & $1.35 \mathrm{e}-2$ & $9.93 \mathrm{e}-3$ & $8.47 \mathrm{e}-3$ & $8.95 \mathrm{e}-3$ \\
0.8 & $4.38 \mathrm{e}-1$ & $6.40 \mathrm{e}-3$ & $1.13 \mathrm{e}-2$ & $9.27 \mathrm{e}-3$ & $1.89 \mathrm{e}-2$ \\
0.9 & $4.85 \mathrm{e}-1$ & $1.51 \mathrm{e}-2$ & $1.28 \mathrm{e}-2$ & $2.04 \mathrm{e}-1$ & $2.04 \mathrm{e}-1$ \\
\hline
\end{tabular}

Table 2

Convergence of path-following method. For each step $k$, the parameter $\beta(k)$, number it $(k)$ of SSN iterations, and $\mathrm{L}^{2}$-error $e(k)$ are shown.

\begin{tabular}{ccccccccccc}
\hline$\beta(k)$ & $1.00 \mathrm{e} 0$ & $5.00 \mathrm{e}-1$ & $2.50 \mathrm{e}-1$ & $1.25 \mathrm{e}-1$ & $6.25 \mathrm{e}-2$ & $3.12 \mathrm{e}-2$ & $1.56 \mathrm{e}-2$ & $7.81 \mathrm{e}-3$ & $3.91 \mathrm{e}-3$ & $1.95 \mathrm{e}-3$ \\
\hline$i t(k)$ & 6 & 4 & 4 & 3 & 3 & 3 & 3 & 3 & 3 & 3 \\
$e(k)$ & $1.83 \mathrm{e}-1$ & $1.33 \mathrm{e}-1$ & $1.08 \mathrm{e}-1$ & $8.77 \mathrm{e}-2$ & $7.17 \mathrm{e}-2$ & $5.99 \mathrm{e}-2$ & $4.63 \mathrm{e}-2$ & $3.43 \mathrm{e}-2$ & $2.63 \mathrm{e}-2$ & $2.11 \mathrm{e}-2$ \\
\hline \hline$\beta(k)$ & $9.77 \mathrm{e}-4$ & $4.88 \mathrm{e}-4$ & $2.44 \mathrm{e}-4$ & $1.22 \mathrm{e}-4$ & $6.10 \mathrm{e}-5$ & $3.05 \mathrm{e}-5$ & $1.53 \mathrm{e}-5$ & $7.63 \mathrm{e}-6$ & $3.81 \mathrm{e}-6$ & $1.91 \mathrm{e}-6$ \\
\hline$i t(k)$ & 3 & 3 & 3 & 3 & 3 & 3 & 3 & 3 & 3 & 4 \\
$e(k)$ & $1.70 \mathrm{e}-2$ & $1.33 \mathrm{e}-2$ & $1.03 \mathrm{e}-2$ & $8.06 \mathrm{e}-3$ & $6.29 \mathrm{e}-3$ & $4.90 \mathrm{e}-3$ & $3.80 \mathrm{e}-3$ & $2.94 \mathrm{e}-3$ & $2.31 \mathrm{e}-3$ & $1.85 \mathrm{e}-3$ \\
\hline \hline$\beta(k)$ & $9.54 \mathrm{e}-7$ & $4.77 \mathrm{e}-7$ & $2.38 \mathrm{e}-7$ & $1.19 \mathrm{e}-7$ & $5.96 \mathrm{e}-8$ & $2.98 \mathrm{e}-8$ & $1.49 \mathrm{e}-8$ & $7.45 \mathrm{e}-9$ & $3.73 \mathrm{e}-9$ & $1.86 \mathrm{e}-9$ \\
\hline$i t(k)$ & 4 & 5 & 5 & 9 & 14 & 20 & 20 & 20 & 20 & 20 \\
$e(k)$ & $1.51 \mathrm{e}-3$ & $1.28 \mathrm{e}-3$ & $1.12 \mathrm{e}-3$ & $1.00 \mathrm{e}-3$ & $9.32 \mathrm{e}-4$ & $9.08 \mathrm{e}-4$ & $8.90 \mathrm{e}-4$ & $8.66 \mathrm{e}-4$ & $8.65 \mathrm{e}-4$ & $8.66 \mathrm{e}-4$ \\
\hline
\end{tabular}

Table 3

Convergence behavior of the SSN method (for fixed $\alpha, \beta$ ) for the $1 d$ inverse potential problem. Shown are the problem size $N$, the number $n(k)$ of elements that changed between active and inactive sets and residual norm $r(k) \equiv\|F(u)\|_{\mathrm{L}^{2}}$ after each iteration $k$.

\begin{tabular}{ccccccc}
\hline$N$ & $k$ & 1 & 2 & 3 & 4 & 5 \\
\hline \multirow{2}{*}{101} & $n(k)$ & 88 & 0 & 0 & 0 & 0 \\
& $r(k)$ & $4.40 \mathrm{e}-2$ & $1.51 \mathrm{e}-2$ & $8.62 \mathrm{e}-4$ & $6.58 \mathrm{e}-6$ & $2.86 \mathrm{e}-10$ \\
\hline \multirow{2}{*}{1001} & $n(k)$ & 791 & 6 & 5 & 0 & 0 \\
& $r(k)$ & $1.23 \mathrm{e}-1$ & $1.78 \mathrm{e}-2$ & $2.30 \mathrm{e}-3$ & $3.53 \mathrm{e}-5$ & $9.99 \mathrm{e}-9$ \\
\hline \multirow{2}{*}{10001} & $n(k)$ & 7803 & 91 & 16 & 1 & 0 \\
& $r(k)$ & $1.21 \mathrm{e}-1$ & $1.67 \mathrm{e}-2$ & $1.68 \mathrm{e}-3$ & $1.90 \mathrm{e}-5$ & $2.47 \mathrm{e}-9$ \\
\hline
\end{tabular}




\section{Table 4}

Computing times (in seconds) for SSN method $\left(t_{\mathrm{s}}\right)$ and fixed point iteration $\left(t_{\mathrm{b}}\right)$ and $\mathrm{L}^{2}$-error e for the $1 d$ inverse potential problem. Shown are the problem size $N$, the mean $\left(\left\{t_{\mathrm{s}}, t_{\mathrm{b}}, e\right\}_{\mathrm{m}}\right)$, and the standard deviation $\left(\left\{t_{\mathrm{s}}, t_{\mathrm{b}}, e\right\}_{\mathrm{s}}\right)$ over 10 noise realizations.

\begin{tabular}{ccccccccc}
\hline$N$ & 100 & 200 & 400 & 800 & 1600 & 3200 & 6400 & 12800 \\
\hline$t_{\mathrm{s}, \mathrm{m}}$ & 1.25 & 1.75 & 5.28 & 12.09 & 19.40 & 29.66 & 55.33 & 107.87 \\
$t_{\mathrm{s}, \mathrm{s}}$ & 0.48 & 0.45 & 3.31 & 4.57 & 7.22 & 4.45 & 11.44 & 25.92 \\
\hline$t_{\mathrm{b}, \mathrm{m}}$ & 7.12 & 9.63 & 14.42 & 39.04 & 54.19 & 80.30 & 131.72 & 234.00 \\
$t_{\mathrm{b}, \mathrm{s}}$ & 3.42 & 6.21 & 7.63 & 17.14 & 16.79 & 17.25 & 38.08 & 75.21 \\
\hline$e_{\mathrm{m}}$ & $8.98 \mathrm{e}-1$ & $1.51 \mathrm{e}+0$ & $2.88 \mathrm{e}-3$ & $9.17 \mathrm{e}-4$ & $6.22 \mathrm{e}-4$ & $3.52 \mathrm{e}-4$ & $2.76 \mathrm{e}-4$ & $2.78 \mathrm{e}-4$ \\
$e_{\mathrm{s}}$ & $2.46 \mathrm{e}+0$ & $3.16 \mathrm{e}+0$ & $2.05 \mathrm{e}-3$ & $5.76 \mathrm{e}-4$ & $6.83 \mathrm{e}-4$ & $9.36 \mathrm{e}-5$ & $4.29 \mathrm{e}-5$ & $6.93 \mathrm{e}-5$ \\
\hline
\end{tabular}

Table 5

Comparison of the balancing principle $\left(\alpha_{\mathrm{b}}, e_{\mathrm{b}}\right)$ with the sampling-based optimal choice $\left(\alpha_{\mathrm{o}}, e_{\mathrm{o}}\right)$ for the inverse Robin coefficient problem.

\begin{tabular}{cccccc}
\hline$r$ & $\delta$ & $\alpha_{\mathrm{o}}$ & $\alpha_{\mathrm{b}}$ & $e_{\mathrm{o}}$ & $e_{\mathrm{b}}$ \\
\hline 0.1 & $1.31 \mathrm{e}+0$ & $1.40 \mathrm{e}-1$ & $3.38 \mathrm{e}-2$ & $1.15 \mathrm{e}-5$ & $4.10 \mathrm{e}-5$ \\
0.2 & $2.19 \mathrm{e}+0$ & $1.07 \mathrm{e}-1$ & $5.62 \mathrm{e}-2$ & $4.03 \mathrm{e}-6$ & $1.46 \mathrm{e}-5$ \\
0.3 & $3.41 \mathrm{e}+0$ & $2.45 \mathrm{e}-1$ & $8.76 \mathrm{e}-2$ & $8.63 \mathrm{e}-4$ & $1.22 \mathrm{e}-3$ \\
0.4 & $5.30 \mathrm{e}+0$ & $6.27 \mathrm{e}-1$ & $1.36 \mathrm{e}-1$ & $2.64 \mathrm{e}-3$ & $5.40 \mathrm{e}-3$ \\
0.5 & $6.00 \mathrm{e}+0$ & $5.01 \mathrm{e}-1$ & $1.54 \mathrm{e}-1$ & $4.10 \mathrm{e}-4$ & $1.53 \mathrm{e}-3$ \\
0.6 & $7.31 \mathrm{e}+0$ & $4.41 \mathrm{e}-1$ & $1.88 \mathrm{e}-1$ & $3.72 \mathrm{e}-2$ & $6.30 \mathrm{e}-2$ \\
0.7 & $9.13 \mathrm{e}+0$ & $3.40 \mathrm{e}-1$ & $2.35 \mathrm{e}-1$ & $6.07 \mathrm{e}-3$ & $6.36 \mathrm{e}-3$ \\
0.8 & $9.79 \mathrm{e}+0$ & $2.53 \mathrm{e}-1$ & $2.53 \mathrm{e}-1$ & $6.59 \mathrm{e}-2$ & $6.59 \mathrm{e}-2$ \\
0.9 & $1.16 \mathrm{e}+1$ & $6.00 \mathrm{e}-1$ & $3.16 \mathrm{e}-1$ & $3.02 \mathrm{e}-1$ & $3.37 \mathrm{e}-1$ \\
\hline
\end{tabular}

Table 6

Comparison of the balancing principle $\left(\alpha_{\mathrm{b}}, e_{\mathrm{b}}\right)$ with the sampling-based optimal choice $\left(\alpha_{\mathrm{o}}, e_{\mathrm{o}}\right)$ for the $1 d$ inverse diffusion coefficient problem.

\begin{tabular}{cccccc}
\hline$r$ & $\delta$ & $\alpha_{\mathrm{o}}$ & $\alpha_{\mathrm{b}}$ & $e_{\mathrm{o}}$ & $e_{\mathrm{b}}$ \\
\hline 0.1 & $2.08 \mathrm{e}-2$ & $9.20 \mathrm{e}-5$ & $1.52 \mathrm{e}-4$ & $2.34 \mathrm{e}-5$ & $2.71 \mathrm{e}-5$ \\
0.2 & $3.81 \mathrm{e}-2$ & $1.43 \mathrm{e}-4$ & $2.77 \mathrm{e}-4$ & $2.17 \mathrm{e}-5$ & $2.28 \mathrm{e}-5$ \\
0.3 & $5.82 \mathrm{e}-2$ & $3.41 \mathrm{e}-4$ & $3.99 \mathrm{e}-4$ & $2.68 \mathrm{e}-5$ & $3.98 \mathrm{e}-5$ \\
0.4 & $8.54 \mathrm{e}-2$ & $2.11 \mathrm{e}-4$ & $5.71 \mathrm{e}-4$ & $2.59 \mathrm{e}-5$ & $3.84 \mathrm{e}-5$ \\
0.5 & $9.45 \mathrm{e}-2$ & $3.83 \mathrm{e}-4$ & $5.98 \mathrm{e}-4$ & $3.56 \mathrm{e}-5$ & $4.28 \mathrm{e}-5$ \\
0.6 & $1.22 \mathrm{e}-1$ & $1.28 \mathrm{e}-3$ & $7.45 \mathrm{e}-4$ & $2.82 \mathrm{e}-4$ & $3.60 \mathrm{e}-4$ \\
0.7 & $1.42 \mathrm{e}-1$ & $2.04 \mathrm{e}-3$ & $8.35 \mathrm{e}-4$ & $8.01 \mathrm{e}-4$ & $1.31 \mathrm{e}-3$ \\
0.8 & $1.55 \mathrm{e}-1$ & $1.66 \mathrm{e}-3$ & $8.71 \mathrm{e}-4$ & $4.82 \mathrm{e}-4$ & $6.52 \mathrm{e}-4$ \\
0.9 & $1.80 \mathrm{e}-1$ & $4.33 \mathrm{e}-3$ & $9.42 \mathrm{e}-4$ & $2.11 \mathrm{e}-3$ & $6.49 \mathrm{e}-3$ \\
\hline
\end{tabular}


Table 7

Computing times (in seconds) for the SSN method $\left(t_{\mathrm{s}}\right)$ and fixed point iteration $\left(t_{\mathrm{b}}\right)$ and $\mathrm{L}^{2}$-error e for the $1 d$ inverse diffusion coefficient problem. Shown are the problem size $N$, the mean $\left(\left\{t_{\mathrm{s}}, t_{\mathrm{b}}, e\right\}_{\mathrm{m}}\right)$, and the standard deviation $\left(\left\{t_{\mathrm{s}}, t_{\mathrm{b}}, e\right\}_{\mathrm{s}}\right)$ over 10 noise realizations.

\begin{tabular}{ccccccccc}
\hline$N$ & 100 & 200 & 400 & 800 & 1600 & 3200 & 6400 & 12800 \\
\hline$t_{\mathrm{s}, \mathrm{m}}$ & 0.70 & 2.19 & 6.49 & 11.46 & 25.48 & 55.34 & 82.38 & 167.71 \\
$t_{\mathrm{s}, \mathrm{s}}$ & 0.46 & 1.65 & 0.91 & 2.80 & 5.34 & 17.19 & 23.07 & 31.01 \\
\hline$t_{\mathrm{b}, \mathrm{m}}$ & 4.12 & 8.68 & 19.27 & 27.59 & 52.45 & 97.32 & 154.46 & 332.96 \\
$t_{\mathrm{b}, \mathrm{s}}$ & 2.51 & 3.71 & 2.20 & 6.28 & 9.14 & 21.96 & 22.59 & 39.17 \\
\hline$e_{\mathrm{m}}$ & $2.17 \mathrm{e}-1$ & $8.03 \mathrm{e}-2$ & $4.94 \mathrm{e}-5$ & $3.20 \mathrm{e}-5$ & $2.89 \mathrm{e}-5$ & $3.33 \mathrm{e}-5$ & $3.39 \mathrm{e}-5$ & $3.08 \mathrm{e}-5$ \\
$e_{\mathrm{s}}$ & $2.05 \mathrm{e}-1$ & $1.53 \mathrm{e}-1$ & $2.66 \mathrm{e}-5$ & $5.86 \mathrm{e}-6$ & $4.89 \mathrm{e}-6$ & $6.74 \mathrm{e}-6$ & $5.42 \mathrm{e}-6$ & $3.25 \mathrm{e}-6$ \\
\hline
\end{tabular}

Acknowledgments. The authors are grateful to the referees for their constructive comments which have led to an improved presentation.

\section{REFERENCES}

[1] R. A. Adams And J. J. F. Fournier, Sobolev Spaces, 2nd ed., Elsevier/Academic Press, Amsterdam, 2003.

[2] W. K. AllaRd, Total variation regularization for image denoising. I. Geometric theory, SIAM J. Math. Anal., 39 (2007/08), pp. 1150-1190.

[3] H. T. BAnks And K. Kunisch, Estimation Techniques for Distributed Parameter Systems, Birkhäuser, Boston, 1989.

[4] S. L. Bernstein, M. L. Burrows, J. E. Evans, A. S. Griffiths, D. A. Mcneill, C. W. Niessen, I. Richer, D. P. White, And D. K. Willim, Long-range communications at extremely low frequencies, Proc. IEEE, 62 (1974), pp. 292-312.

[5] N. Bissantz, T. Hohage, A. Munk, And F. Ruymgant, Convergence rates of general regularization methods for statistical inverse problems and applications, SIAM J. Numer. Anal., 45 (2007), pp. 26102636 .

[6] A. C. BoviK, Handbook of Image and Video Processing (Communications, Networking and Multimedia), Academic Press, Orlando, FL, 2005.

[7] M. Burger And S. Osher, Convergence rates of convex variational regularization, Inverse Problems, 20 (2004), pp. 1411-1421.

[8] S. ChaAbane, J. Ferchichi, And K. Kunisch, Differentiability properties of the $L^{1}$-tracking functional and application to the Robin inverse problem, Inverse Problems, 20 (2004), pp. 1083-1097.

[9] T. F. Chan And S. EsEdō̄LU, Aspects of total variation regularized $L^{1}$ function approximation, SIAM J. Appl. Math., 65 (2005), pp. 1817-1837.

[10] S. S. Chen, D. L. Donoho, And M. A. SAunders, Atomic decomposition by basis pursuit, SIAM J. Sci. Comput., 20 (1998), pp. 33-61.

[11] X. Chen, Z. NAshed, And L. QI, Smoothing methods and semismooth methods for nondifferentiable operator equations, SIAM J. Numer. Anal., 38 (2000), pp. 1200-1216.

[12] Z. Chen AND J. Zou, An augmented Lagrangian method for identifying discontinuous parameters in elliptic systems, SIAM J. Control Optim., 37 (1999), pp. 892-910.

[13] F. H. Clarke, Optimization and Nonsmooth Analysis, 2nd ed., Classics Appl. Math. 5, SIAM, Philadelphia, 1990.

[14] C. Clason, B. Jin, And K. Kunisch, A duality-based splitting method for $\ell^{1}-T V$ image restoration with automatic regularization parameter choice, SIAM J. Sci. Comput., 32 (2010), pp. 1484-1505.

[15] C. Clason, B. Jin, And K. Kunisch, A semismooth Newton method for $L^{1}$ data fitting with automatic choice of regularization parameters and noise calibration, SIAM J. Imaging Sci., 3 (2010), pp. 199-231. 
[16] Y. Dong, M. Hintermüller, And M. Neri, An efficient primal-dual method for $\ell^{1}$ TV image restoration, SIAM J. Imaging Sci., 2 (2009), pp. 1168-1189.

[17] V. Duval, J.-F. Aujol, And Y. Gousseau, The TVL1 model: A geometric point of view, Multiscale Model. Simul., 8 (2009), pp. 154-189.

[18] I. Ekeland AND R. TÉmAM, Convex analysis and variational problems, Classics Appl. Math. 28, SIAM, Philadelphia, 1999.

[19] H. W. Engl, M. Hanke, and A. Neubauer, Regularization of Inverse Problems, Kluwer, Dordrecht, The Netherlands, 1996.

[20] H. W. ENGL, K. Kunisch, AND A. Neubauer, Convergence rates for Tikhonov regularisation of nonlinear ill-posed problems, Inverse Problems, 5 (1989), pp. 523-540.

[21] L. C. Evans and R. F. Gariepy, Measure Theory and Fine Properties of Functions, Stud. Adv. Math., CRC Press, Boca Raton, FL, 1992.

[22] A. Gelman, J. B. Carlin, H. S. Stern, and D. B. Rubin, Bayesian Data Analysis, 2nd ed., Chapman \& Hall/CRC, Boca Raton, FL, 2004.

[23] M. Hintermüller, K. Ito, AND K. Kunisch, The primal-dual active set strategy as a semismooth Newton method, SIAM J. Optim., 13 (2002), pp. 865-888 (2003).

[24] M. Hintermüller And M. M. Rincon-CAmacho, Expected absolute value estimators for a spatially adapted regularization parameter choice rule in $L^{1}-T V$-based image restoration, Inverse Problems, 26 (2010), p. 085005.

[25] M. Hintermüller And M. Ulbrich, A mesh-independence result for semismooth Newton methods, Math. Program., 101 (2004), pp. 151-184.

[26] B. Hofmann, B. Kaltenbacher, C. Pöschl, and O. Scherzer, A convergence rates result for Tikhonov regularization in Banach spaces with non-smooth operators, Inverse Problems, 23 (2007), pp. 987-1010.

[27] D. Hsu, S. M. KakAde, And T. Zhang, Robust matrix decomposition with sparse corruptions, IEEE Trans. Inform. Theory, 57 (2011), pp. 7221-7234.

[28] P. J. Huber, Robust Statistics, John Wiley \& Sons, New York, 1981.

[29] K. Iто AND B. Jin, A new approach to nonlinear constrained Tikhonov regularization, Inverse Problems, 27 (2011), p. 105005.

[30] K. Ito, B. Jin, AND T. TAKeuchi, A regularization parameter for nonsmooth Tikhonov regularization, SIAM J. Sci. Comput., 33 (2011), pp. 1415-1438.

[31] K. Ito And K. Kunisch, Lagrange Multiplier Approach to Variational Problems and Applications, Adv. Des. Control 15, SIAM, Philadelphia, 2008.

[32] B. Jin And J. Zou, Iterative parameter choice by discrepancy principle, IMA J. Numer. Anal., doi: 10.1093/imanum/drr051.

[33] B. JIN AND J. Zou, Numerical estimation of the Robin coefficient in a stationary diffusion equation, IMA J. Numer. Anal., 30 (2010), pp. 677-701.

[34] T. KärkKäInen, K. Kunisch, And K. Majava, Denoising of smooth images using $L^{1}$-fitting, Computing, 74 (2005), pp. 353-376.

[35] A. KRÖNER AND B. VeXler, A priori error estimates for elliptic optimal control problems with a bilinear state equation, J. Comput. Appl. Math., 230 (2009), pp. 781-802.

[36] B. Kummer, Generalized Newton and NCP-methods: Convergence, regularity, actions, Discuss. Math. Differ. Incl. Control Optim., 20 (2000), pp. 209-244.

[37] M. Lassas, E. Saksman, And S. Siltanen, Discretization-invariant Bayesian inversion and Besov space priors, Inverse Problems Imaging, 3 (2009), pp. 87-122.

[38] N. G. MEYERs, An $L^{p}$-estimate for the gradient of solutions of second order elliptic divergence equations, Ann. Scuola Norm. Sup. Pisa (3), 17 (1963), pp. 189-206.

[39] V. A. Morozov, On the solution of functional equations by the method of regularization, Soviet Math. Dokl., 7 (1966), pp. 414-417.

[40] C. Pöschl, An overview on convergence rates for Tikhonov regularization methods for non-linear operators, J. Inverse Ill-Posed Probl., 17 (2009), pp. 77-83.

[41] A. Schiela, A simplified approach to semismooth Newton methods in function space, SIAM J. Optim., 19 (2008), pp. 1417-1432.

[42] S. She AND A. B. Owen, Outlier detection using nonconvex penalized regression, J. Amer. Statist. Assoc., 106 (2011), pp. 626-639. 
[43] S. Stojanovic, Optimal damping control and nonlinear elliptic systems, SIAM J. Control Optim., 29 (1991), pp. 594-608.

[44] R. Tibshirani, Regression shrinkage and selection via the lasso, J. Roy. Statist. Soc. Ser. B, 58 (1996), pp. $267-288$

[45] F. Tröltzsch, Optimal Control of Partial Differential Equations: Theory, Methods and Applications, American Mathematical Society, Providence, RI, 2010.

[46] M. Ulbrich, Semismooth Newton methods for operator equations in function spaces, SIAM J. Optim., 13 (2002), pp. 805-842 (2003).

[47] M. Yамамото And J. Zou, Simultaneous reconstruction of the initial temperature and heat radiative coefficient, Inverse Problems, 17 (2001), pp. 1181-1202.

[48] J. YANG, Y. Zhang, AND W. YIN, An efficient TVL1 algorithm for deblurring multichannel images corrupted by impulsive noise, SIAM J. Sci. Comput., 31 (2009), pp. 2842-2865.

[49] W. W.-G. YEH, Review of parameter identification procedures in groundwater hydrology: The inverse problem, Water Resource Res., 22 (1986), pp. 95-108.

[50] W. Yin, D. Goldfarb, AND S. Osher, The total variation regularized $L^{1}$ model for multiscale decomposition, Multiscale Model. Simul., 6 (2007), pp. 190-211. 